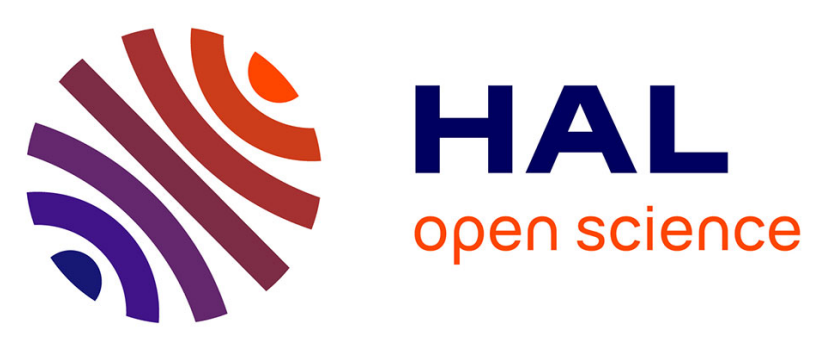

\title{
Bis-14-membered ring diketal diamines: Synthesis and evaluation of their anti-HIV and anti-tumoral activities.
}

Radouane Affani, Franck Pélissier, Anne-Marie Aubertin, Denise Dugat

\section{To cite this version:}

Radouane Affani, Franck Pélissier, Anne-Marie Aubertin, Denise Dugat. Bis-14-membered ring diketal diamines: Synthesis and evaluation of their anti-HIV and anti-tumoral activities.. European Journal of Medicinal Chemistry, 2009, 44, epub ahead of print. 10.1016/j.ejmech.2009.03.008 . hal-00384465

\section{HAL Id: hal-00384465 \\ https://hal.science/hal-00384465}

Submitted on 15 May 2009

HAL is a multi-disciplinary open access archive for the deposit and dissemination of scientific research documents, whether they are published or not. The documents may come from teaching and research institutions in France or abroad, or from public or private research centers.
L'archive ouverte pluridisciplinaire HAL, est destinée au dépôt et à la diffusion de documents scientifiques de niveau recherche, publiés ou non, émanant des établissements d'enseignement et de recherche français ou étrangers, des laboratoires publics ou privés. 


\title{
Bis-14-membered ring diketal diamines: Synthesis and evaluation of their anti-HIV and anti-tumoral activities
}

\author{
Radouane Affani ${ }^{\mathrm{a}}$, Franck Pélissier ${ }^{\mathrm{a}}$, Anne-Marie Aubertin ${ }^{\mathrm{b}}$ and Denise Dugat ${ }^{\mathrm{a}}$ \\ ${ }^{a}$ Laboratoire SEESIB (Synthèse Et Etude de Systèmes à Intérêt Biologique), UMR CNRS 6504, \\ Université Blaise Pascal de Clermont-Ferrand, 24 Avenue des Landais, 63177 Aubière, France \\ ${ }^{\mathrm{b}}$ Institut de Virologie, INSERM U778, Université Louis Pasteur, 67000 Strasbourg, France
}

\begin{abstract}
Chiral and achiral macrocyclic bis-diketal diamines, analogs of bicyclam AMD 3100, were synthesized in three steps from the previously obtained 14-membered ring diketal dilactams. Their monoreduction with lithium aluminium hydride gave the corresponding diketal aminolactams. Coupling these with dibromo- $p$ xylene led to xylyl dimer compounds. A second reduction step yielded the expected bis-diketal diamines in the methyl and unsubstituted series. Biological tests on the unreduced and reduced dimers showed both weak anti-HIV and anti-proliferative activities for the bis-diphenyl diketal aminolactam $\mathbf{1 3 b}$, with a mode of action probably different from that of AMD 3100.
\end{abstract}

\section{Introduction}

Macrocyclic saturated polyamines have generated continuous interest because of their physicochemical and biological properties [1]. Among these derivatives, bicyclams form a new class of compounds that exhibit anti-viral [1b, 2-7] and anti-tumoral [7-9] activities as antagonists of the chemokine co-receptor CXCR4 of the cell membrane [3-9]. As anti-HIV-1 agents, they interfere at the virus-cell fusion step in blocking the interaction between CXCR4 and the glycoproteins gp 120 of the viral envelope, i.e. the entry of the virus into the host cell, and thereby its replication [3-7]. As anti-tumoral agents, they block the binding of CXCR4 with its natural ligand, the chemokine C-X-C, SDF-1 $\alpha$ (stromal cell-derived factor), and hence inhibit growth, migration and proliferation of cancerous cells [7-9]. One of these compounds, the xylyl bicyclam AMD 3100, several synthesis of which have been reported [2b, 3c,10], has so far proved to be the most specific CXCR4 antagonist with a nanomolar binding affinity [2a, 3a, 4c, 5-7]. However, this molecule presents poor oral bioavailability due to its highly polar nature [5-6], and possible cardiac side effects disclosed after Phase II trials [6]. In this light, novel chemical analogs of this bioactive agent were likely to be of interest.

In continuation of our work on the synthesis, conformational analysis and binding properties of new macrocyclic 14-membered ring diketal dilactams [11], and diketal diamines [12], we report the synthesis of substituted bis-dioxygenated analogs of bicyclam AMD 3100 (Fig. 1) and the evaluation of their antiviral and anti-tumoral activities. 

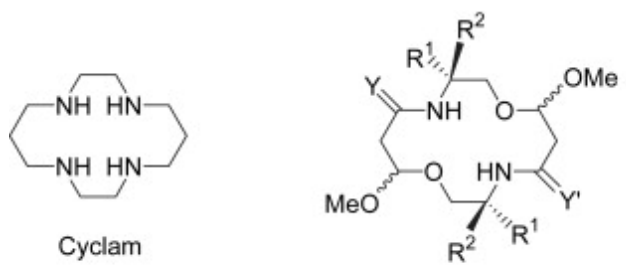

Diketal dilactams $\quad: Y=Y^{\prime}=0$

Diketal diamines $\quad: Y=Y^{\prime}=\mathrm{H}_{2}$

Diketal aminolactams : $Y=O ; Y^{\prime}=\mathrm{H}_{2}$

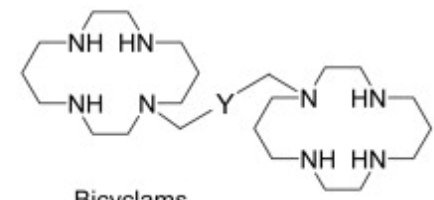

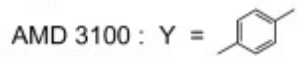

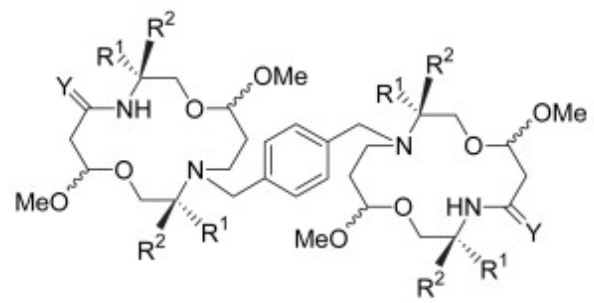

Bis-diketal aminolactams : $Y=0$

Bis-diketal diamines $\quad: \mathrm{Y}=\mathrm{H}_{2}$

Fig. 1. General structure of cyclam, diketal dilactams, diketal diamines, diketal aminolactams, bicyclams, bis-diketal dilactams, and bis-diketal diamines.

\section{Chemistry}

\subsection{Synthesis}

Bis-diketal diamines were targeted by a coupling reaction of diketal aminolactams (Fig. 1), previously obtained as minor products in the synthesis of diketal diamines by reduction of the corresponding diketal dilactams [12].

\subsubsection{Synthesis of diketal aminolactams}

The study was conducted on the two major isomers $\mathbf{b}$ (trans-OMe) and $\mathbf{c}$ (cis-OMe) [13] of the three already developed series of diketal dilactams $\left(\mathbf{1}: R^{1}=P h, R^{2}=H ; 2: R^{1}=H, R^{2}=M e ; 3: R^{1}=R^{2}=H\right)$, using lithium aluminium hydride (10 equiv.) as reducing agent [12],. However, some modifications were made to the previously described reaction conditions to invert the ratio of singly reduced compounds 4-6 to doubly reduced compounds 7-9 (Fig. 2, Table 1). As previously, small amounts of $\mathrm{NEt}_{3} \mathrm{were}$ added to minimize the effect of an elimination side reaction leading to unsaturated ketal aminolactams 10-12 [12]. 

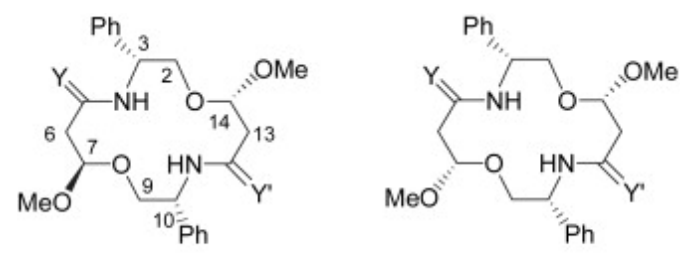

$$
\begin{array}{lll}
\text { 4b } & Y=O ; Y^{\prime}=\mathrm{H}_{2} & \text { 4c } \\
\text { 7b } & Y=Y^{\prime}=\mathrm{H}_{2} & \text { 7c }
\end{array}
$$
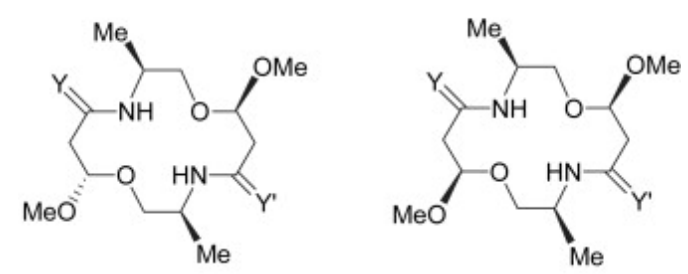

$$
\begin{array}{lll}
\text { 2b } & Y=Y^{\prime}=O & \text { 2c } \\
\text { 5b } & Y=O ; Y^{\prime}=H_{2} & \text { 5c } \\
\text { 8b } & Y=Y^{\prime}=H_{2} & \text { 8c }
\end{array}
$$
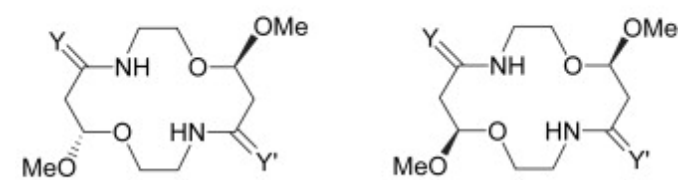

$$
\begin{array}{lll}
\text { 3b } & Y=Y^{\prime}=O & 3 c \\
\text { 6b } & Y=O ; Y^{\prime}=H_{2} & 6 c \\
\text { 9b } & Y=Y^{\prime}=H_{2} & 9 c
\end{array}
$$

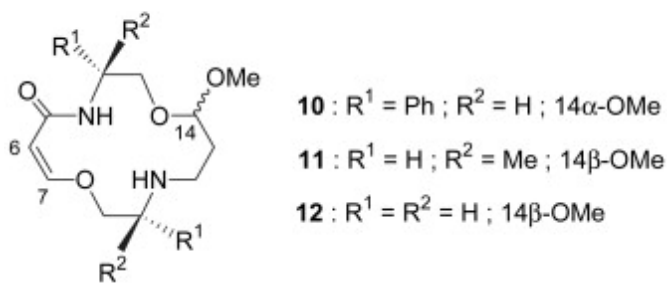

Fig. 2. Structure and stereochemistry of compounds $\mathbf{1 b}, \mathbf{c}-\mathbf{9 b}, \mathbf{c}, \mathbf{1 0}-\mathbf{1 2}$.

\section{Table 1.}

Reduction of diketal dilactams $\mathbf{1 b}, \mathbf{c}, \mathbf{2} \mathbf{b}, \mathbf{c}$ and $\mathbf{3} \mathbf{b}, \mathbf{c}$ by LAH (10 equiv) in THF, and in the presence of small amounts of $\mathrm{NEt}_{3}(0.1-0.2$ equiv.).

\begin{tabular}{|l|l|l|l|l|l|}
\hline Entrv & Substrate $(\mathbf{R})$ & $\left.\mathbf{c}_{\text {suhstrate }} \mathbf{( 1 0}^{-\mathbf{3}} \mathbf{M}\right)$ & Time (h) & Conv. (\%) $^{\mathbf{d}}$ & Results $^{\mathbf{e}} \mathbf{( \% )}$ \\
\hline $1^{\underline{a}}$ & $\mathbf{1 c}(\mathrm{Ph})$ & 17 & 9 & 90 & $\mathbf{4 c}(16), \mathbf{7 c}(21), \mathbf{1 0}(14)$ \\
\hline $2^{\underline{b}}$ & $\mathbf{1 c}(\mathrm{Ph})$ & 25 & 11 & 95 & $\mathbf{4 c}(8), \mathbf{7 c}(30), \mathbf{1 0}(8)$ \\
\hline 3 & $\mathbf{1 c}(\mathrm{Ph})$ & 8 & 9 & 84 & $\mathbf{4 c}(35), \mathbf{7 c}(10), \mathbf{1 0}(8)$ \\
\hline 4 & $\mathbf{1 b}(\mathrm{Ph})$ & 14 & 10 & 85 & $\mathbf{4 b}(48), \mathbf{7 b}(4)$ \\
\hline 5 & $\mathbf{2 b}(\mathrm{Me})$ & 12 & 18 & 93 & $\mathbf{5 b}(21), \mathbf{8 b}(20), \mathbf{1 1}(2)$ \\
\hline 6 & $\mathbf{2 b}(\mathrm{Me})$ & 8 & 18 & 88 & $\mathbf{5 b}(28), \mathbf{8 b}(12), \mathbf{1 1}(2)$ \\
\hline 7 & $\mathbf{2 c}(\mathrm{Me})$ & 10 & 20 & 85 & $\mathbf{8 c}(17)$ \\
\hline 8 & $\mathbf{2 c}(\mathrm{Me})$ & 5 & 20 & 85 & $\mathbf{5 c}(1), \mathbf{8 c}(12)$ \\
\hline $9 \underline{c}$ & $\mathbf{2 c}(\mathrm{Me})$ & 4 & 9 & 60 & $\mathbf{5 c}(12), \mathbf{8 c}(22), \mathbf{1 1}(1)$ \\
\hline 10 & $\mathbf{3 b}(\mathrm{H})$ & 5 & 9 & 78 & $\mathbf{6 b}(27) .9 b(16) .12(2)$ \\
\hline
\end{tabular}




\begin{tabular}{|l|l|l|l|l|l|}
\hline Entrv & Substrate (R) & $\left.\boldsymbol{C}_{\text {cuhstrata }} \mathbf{( 1 0}^{-\mathbf{3}} \mathbf{M}\right)$ & Time (h) & Conv. (\%) $^{\mathbf{d}}$ & Results $^{\mathbf{e}} \mathbf{( \% )}$ \\
\hline 11 & $\mathbf{3 c}(\mathrm{H})$ & 3 & 10 & 70 & 6c (29), 9c (14) \\
\hline
\end{tabular}

${ }^{\text {a }}$ Absence of $\mathrm{NEt}_{3}$.

${ }^{\mathrm{b}}$ Molar ratio of $\mathrm{NEt}_{3} /$ substrate $=0.4 / 1$.

${ }^{\mathrm{c}}$ Molar ratio of $\mathrm{LAH} / \mathrm{substrate}=6: 1$.

${ }^{\mathrm{d}}$ Conversion rate based on the amount of recovered starting material.

e Isolated yield by column chromatography; at the end of the process, unidentified polar compounds were invariably isolated in $7-22 \%$ yield, which increased with the reaction time.

Comparison of entries 1 and 2 (Table 1), which recall two earlier experiments carried out from phenyl diketal dilactam 1c [12a], showed that a lower substrate concentration favoured the formation of diketal aminolactam $\mathbf{4 c}$, the yield of which could be optimized in the conditions of entry 3 . Entry 4 reports the optimal formation conditions of both diketal aminolactam $\mathbf{4 b}$ and diketal diamine $\mathbf{7 b}$, the reaction always leading mainly to the mono-reduced compound independently of the concentration [12b]. In the methyl series, a better yield of aminolactam $5 \mathbf{b}$ was obtained in the conditions of entry $6\left(c=8 \times 10^{-3} \mathrm{M}\right)$ compared with the previously reported conditions of entry 5 [12b]. In contrast, the formation of $\mathbf{5 c}$ was only perceptible (yield $12 \%$, conversion rate $60 \%$ ) on decreasing the excess of LAH (6 equiv.) and the reaction time $(9 \mathrm{~h})$ (entry 9 compared with entries 7 and 8 ). In the unsubstituted series the best formation conditions of diketal aminolactams $6 \mathbf{b}$ and $\mathbf{6 c}$ were found at concentrations $5 \times 10^{-3} \mathrm{M}$ and $3 \times 10^{-3} \mathrm{M}$ respectively, using 10 equiv. of LAH (entries 10 and 11).

As previously reported [12], in all the series the reduction occurred with moderate total yields (43-53\% for the selected entries) that were difficult to improve. These results may be explained by the presence of polar compounds (Table 1) probably resulting from complexation between the diketal diamines generated and the $\mathrm{Li}^{+}$cation, which could therefore no longer catalyse the reaction [14].

\subsubsection{Synthesis of bis-diketal aminolactams}

The coupling step between diketal aminolactams 4-6 and the $\alpha, \alpha^{\prime}$-dibromo- $p$-xylene was studied from the substrates obtained in appreciable yields, i.e. from $\mathbf{4 b}, \mathbf{c}, \mathbf{5 b}, \mathbf{6 b}, \mathbf{c}$. The reactions were performed with minor variations of literature procedures $[2 b, 3 c, 10]$, using $\mathrm{K}_{2} \mathrm{CO}_{3}$ as basic agent $[2 b, 3 c]$. They led to the expected bis-diketal aminolactams 13-15, obtained in moderate to good yields (37-72\%), and accompanied in both substituted series by small amounts (6-19\%) of bromo ketal aminolactams 16-17, which correspond to single coupling products (Scheme 1).

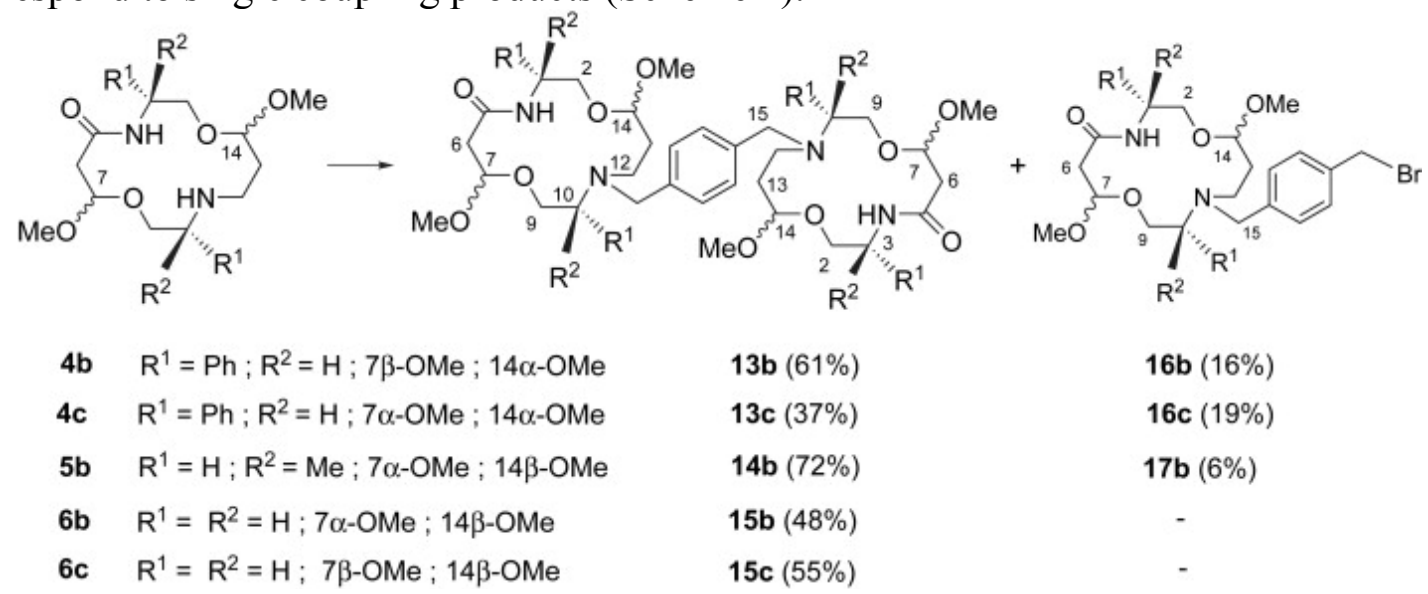

Scheme 1. Reagents and conditions: $\alpha, \alpha^{\prime}$-dibromo- $p$-xylene (0.5-0.6 equiv.), $\mathrm{K}_{2} \mathrm{CO}_{3}$ (1.0-1.2 equiv.), $\mathrm{CH}_{3} \mathrm{CN}\left(c_{\text {substrate }}=50 \times 10^{-3} \mathrm{M}\right), \Delta, 8-10 \mathrm{~h}$.

\subsubsection{Synthesis of bis-diketal diamines}

Due to the moderate yields of $\mathbf{1 3 c}$ and $\mathbf{1 5 b}$, the reduction of bis-diketal aminolactams was studied on only $\mathbf{1 3 b} \mathbf{b} \mathbf{1 4 b}$ and 15c, i.e. on the isomer of each series obtained with the best yield. The reactions were repeated with LAH (10 equiv.) in THF and in the presence of $\mathrm{NEt}_{3}$ (0.02 equiv./LAH). An inverse addition order of the reactants, namely the addition of a commercial THF hydride solution to the substrate solution, was adopted due to the small amounts of starting materials available. 
In the phenyl series, the reduction performed at different substrate concentrations and reaction times led to either an incomplete reaction giving rise to a mixture of singly and doubly reduced bis-macrocycles difficult to separate $\left(c_{\text {substrate }}=30 \times 10^{-3} \mathrm{M}, 10 \mathrm{~h}\right.$ and $\left.40 \times 10^{-3} \mathrm{M}, 9 \mathrm{~h}\right)$, or a major cleavage reaction regenerating diphenyl diketal diamine $7 \mathbf{b}\left(c_{\text {substrate }}=50\right.$ and $\left.60 \times 10^{-3} \mathrm{M}, 8 \mathrm{~h}\right)$.

In contrast, in the methyl and unsaturated series, diketal diamines $\mathbf{1 8 b}$ and $19 \mathrm{c}$ were obtained with $26 \%$ and $22 \%$ yields respectively (conversion rate $100 \%$ ) with the following substrate concentrations and reaction times: $18 \mathbf{b}, c_{\text {substrate }}=100 \times 10^{-3} \mathrm{M}, 9 \mathrm{~h} ; \mathbf{1 9 c}, c_{\text {substrate }}=60 \times 10^{-3} \mathrm{M}, 6 \mathrm{~h}$ (Scheme 2). In both cases, the reduction was also partially accompanied by a benzyl cleavage giving rise to the corresponding diketal diamines $\mathbf{8 b}$ and $\mathbf{9 c}$, in low yield (9\%) compared with the phenyl series.

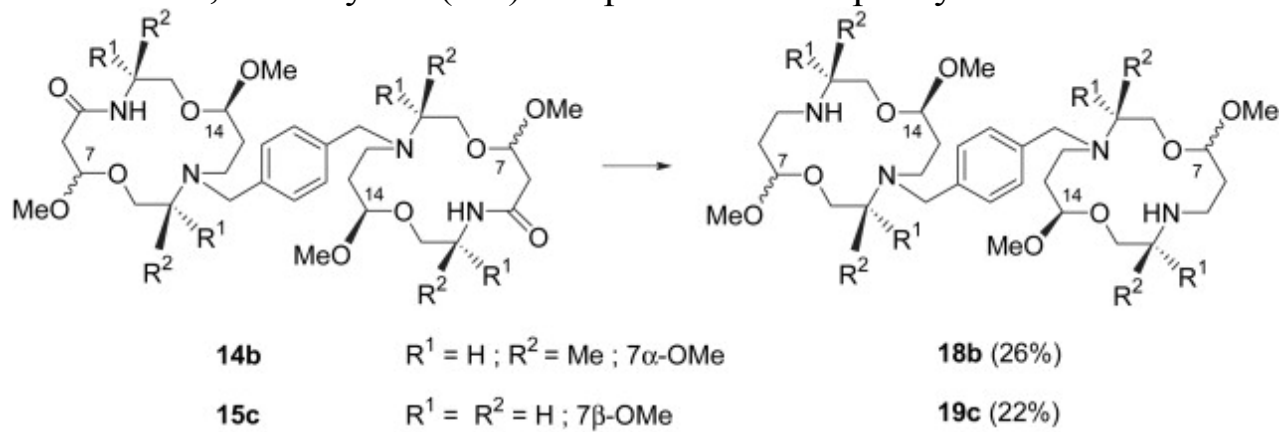

Scheme 2. Reagents and conditions: LAH (10 equiv.), $\mathrm{NEt}_{3} \quad(0.2$ equiv. $)$, THF $\left(c_{\text {substrate }}=60\right.$ $\left.100 \times 10^{-3} \mathrm{M}\right), \Delta, 6-9 \mathrm{~h}$.

In summary, the access to bis-diketal diamines succeeded in the methyl and unsubstituted series but failed in the phenyl series. The difficulties observed in the reduction of the bis-phenyl aminolactam $\mathbf{1 3 b}$, in which the 3,3'-Ph, and 7,7'-OMe groups are in a trans position, are probably due to the hindrance of the two faces of the molecule at the level of the 5,5'-CO carbonyl groups, as previously reported in the reduction of trans-OMe phenyl diketal dilactam $\mathbf{1 b}$ [12b]. In the two successful series, the reduction took place again with moderate total yields (30-35\%) which were difficult to improve as in the formation of compounds $4-9$ above.

\subsection{Characterization}

The structure and stereochemistry of all the derivatives 4-19 were established from IR, MS, 1D NMR $\left[{ }^{1} \mathrm{H},{ }^{13} \mathrm{C}(\mathrm{J}\right.$ MOD) $]$ and 2D NMR [COSY 1H-1H, HSQC (heteronuclear single quantum correlation) and sometimes HMBC (heteronuclear multiple bond correlation)] spectroscopic data, which allowed identification of all the hydrogen and carbon atoms.

The characteristics of the monomer compounds $\mathbf{4 - 1 2}$ have already been reported [12b]. Briefly, (i) in the ${ }^{1} \mathrm{H}$ and ${ }^{13} \mathrm{C}$ NMR spectra, diamines $7 \mathbf{c}, 8 \mathbf{8}, 9 \mathbf{b}, \mathbf{c}$, which all possess a symmetry element $\left(C_{2}\right.$ for $7 \mathbf{c}, 8 \mathbf{8 c}, 9 \mathbf{c}$, center for 9b), show only one signal for each pair of identical groups of the macrocycle, whereas diamines $\mathbf{7 b}$ and $\mathbf{8 b}$ exhibit double signals due to the asymmetry of the two ring chains, and (ii) the reduced carbonyl of trans-OMe aminolactams $\mathbf{4 b}$ and $\mathbf{5 b}$ correspond to the 12-CO carbonyl of chain 2 in which the 14-OMe group and the 3,10-R substituents are in a cis position [12b].

The ${ }^{1} \mathrm{H}$ and ${ }^{13} \mathrm{C}$ NMR spectra of the dimer compounds 13-19 are also simplified due to the presence of a $C_{2}$ axis perpendicular to the aromatic linker.

\section{Biological assays}

Anti-HIV and anti-tumoral tests were performed on the two synthesized bis-diketal diamines $18 \mathbf{b}, \mathbf{1 9 c}$, and on the three bis-diketal aminolactams $\mathbf{1 3 b}, \mathbf{c}, \mathbf{1 5 b}$, not involved in the last reduction step.

\subsection{Anti-HIV activity evaluation}

Since the anti-HIV activity of AMD 3100 appears to be confined to the T-lymphotropic (X4) HIV strains, i.e. those strains that use the CXCR4 receptor to enter their target cells [7b], the effects of the above chemical entities were studied in vitro with two T cell lines, MT-4 and CEM-SS, infected by X4 viral isolates. The activity of a given compound on the replication of HIV-1 was measured by quantification of (i) the inhibition of virus-induced cytopathogenicity in infected MT-4 cells, and (ii) the reverse transcriptase (RT) activity associated with virus particles released from infected CEM-SS cells. 
The $50 \%$ cytotoxic concentration $\left(\mathrm{CC}_{50}\right)$ was evaluated in parallel to the $50 \%$ inhibitory concentration $\left(\mathrm{IC}_{50}\right)$ (Table 2). Among the tested compounds, only the bis-diphenyl diketal aminolactam $\mathbf{1 3 b}$ showed an inhibitory activity in regard to HIV-1 infection in CEM-SS cells, but with lower values than AMD 3100.

Table 2.

Anti-viral activity and cytotoxicity data of bis-diketal aminolactams $\mathbf{1 3 b}, \mathbf{c}, \mathbf{1 5} \mathbf{b}$, and bis-diketal diamines 18b, 19c, evaluated on MT-4 cells and on CEM-SS cells infected with X4 HIV strains (HIV-1IIIB and HIV-1 LAI respectively). ${ }^{\mathrm{a}}$

\begin{tabular}{|l|l|l|l|l|}
\hline Compound (R) & \multicolumn{2}{|l|}{ HIV-1 IIIB/MT4 } & \multicolumn{2}{l|}{ HIV-1 LAI/CEM-SS } \\
\hline & IC $_{\mathbf{5 0}}{ }^{\mathbf{b}}(\boldsymbol{\mu M})$ & $\mathbf{C C}_{\mathbf{5 0}}{ }^{\mathbf{c}}(\boldsymbol{\mu M})$ & IC $_{\mathbf{5 0}} \mathbf{b}(\boldsymbol{\mu M})$ & $\mathbf{C C}_{\mathbf{5 0}}^{\mathbf{c}}(\boldsymbol{\mu M})$ \\
\hline $\mathbf{1 3 b}(\mathrm{Ph})$ & $>\mathrm{CC}_{50}$ & 7.8 & 8.65 & $>10$ \\
\hline $\mathbf{1 3 c}(\mathrm{Ph})$ & $>10$ & $>10$ & $>10$ & $>10$ \\
\hline $\mathbf{1 5 b}(\mathrm{H})$ & $>100$ & $>100$ & $>100$ & $>100$ \\
\hline $\mathbf{1 8 b}(\mathrm{Me})$ & $>100$ & $>100$ & $>100$ & $>100$ \\
\hline $\mathbf{1 9 c}(\mathrm{H})$ & $>\mathrm{CC}_{50}$ & 50 & $>50$ & $>50$ \\
\hline AMD 3100 & 0.41 & 6.5 & 0.32 & $>5$ \\
\hline
\end{tabular}

${ }^{\text {a }}$ All data represent mean values of 2 separate experiments.

${ }^{\mathrm{b}} \mathrm{IC}_{50}: 50 \%$ inhibitory concentration $=$ concentration needed to inhibit $50 \%$ virus replication in vitro.

${ }^{\mathrm{c}} \mathrm{CC}_{50}: 50 \%$ cytotoxic concentration $=$ concentration required to cause $50 \%$ death of uninfected cells.

${ }^{\mathrm{d}}$ Commercial sample purchased from CheMatech.

\subsection{Anti-tumoral activity evaluation [15]}

The in vitro anti-proliferative activities of compounds $13 \mathbf{b}, \mathbf{c}, 15 \mathbf{b}, 18 \mathbf{b}$ and $19 \mathbf{c}$ were evaluated in triplicate towards ten human cell lines [buccal carcinoma (KB), glioblastoma (SF268), myeloid leukaemia (HL60), mammary carcinoma (MCF7 and MCF7R), colon carcinoma (HCT116), pulmonary carcinoma (A549), prostate adenocarcinoma (PC3), ovary adenocarcinoma (OV3) and hepatocarcinoma (HepG2)] using the classic colorimetric MTS test (Table 3). Taxotere was used as a positive control at a drug concentration of $2.5 \times 10^{-10} \mathrm{M}$. The percentage of cell proliferation inhibition was defined as absorbance in experimental wells compared with absorbance in control wells, after subtraction of the blank values. Under the conditions used $\left(10^{-5} \mathrm{M}\right.$ final concentration of tested compounds), the bis-diphenyl diketal aminolactam 13b showed a mild cytotoxic activity of about $50 \%$ towards five cell lines (KB, HL60, MCF7, HCT116 and PC3), i.e. a cytotoxicity corresponding approximately to an $\mathrm{IC}_{50}$ of $\approx 10 \mu \mathrm{M}$.

Table 3.

Percentage cell proliferation inhibition of bis-diketal aminolactams $\mathbf{1 3 b}, \mathbf{c}, \mathbf{1 5 b}$, and bis-diketal diamines $18 b, 19 c$, at a drug concentration of $10^{-5} \mathrm{M}^{\mathrm{a}}$

\begin{tabular}{|l|l|l|l|l|l|l|l|l|l|l|}
\hline Compound & KB & SF268 & HL60 & MCF7 & MCF7R & HCT116 & A549 & PC3 & OV3 & HepG2 \\
\hline $\mathbf{1 3 b}^{\underline{b}}$ & 47 & 27 & 54 & 45 & 14 & 47 & 33 & 51 & 38 & 27 \\
\hline $\mathbf{1 3 c}$ & 12 & 14 & 31 & 25 & 08 & 21 & 17 & 15 & 18 & 00 \\
\hline $\mathbf{1 5 b}$ & 00 & 00 & 10 & 13 & 02 & 07 & 00 & 00 & 00 & 04 \\
\hline $\mathbf{1 8 b}$ & 11 & 05 & 08 & 00 & 04 & 10 & 00 & 09 & 08 & 00 \\
\hline $\mathbf{1 9 c}$ & 00 & 12 & 08 & 08 & 04 & 11 & 00 & 10 & 15 & 00 \\
\hline Taxotere $^{\mathrm{c}}$ & 75 & 37 & 19 & 0 & 0.03 & 24 & 14 & 9 & 22 & 0 \\
\hline
\end{tabular}

${ }^{a}$ All experiments were carried out in triplicate with DMSO as solvent.

${ }^{\mathrm{b}}$ For $13 \mathrm{~b}$, the best observed values $(\approx 50 \%)$ correspond approximately to an $\mathrm{IC}_{50} \approx 10 \mu \mathrm{M}$. 
${ }^{\mathrm{c}}$ Data from Ref. [15].Taxotere was used as a positive control at a drug concentration of $2.5 \times 10^{-10} \mathrm{M}$.

\section{Discussion}

Biological tests, performed on the synthesized bis-macrocyclic compounds $13 \mathbf{b}, \mathbf{c}, \mathbf{1 5 b}, \mathbf{1 8 b}, \mathbf{1 9 c}$ gave an encouraging result for one analog compared with the others: bis-phenyl diketal aminolactam 13b showed both a weak anti-HIV-1 activity and some anti-proliferative effects. The interaction of AMD 3100 with the receptor CXCR4 has been described as probably due to binding of two antiperiplanar NH amine functions of the two cyclam moieties with the free carboxylic acid groups of Asp171 and Asp262 located on respectively transmembrane TM-IV and TM-VI of the receptor [4b,6b,7]. This is not possible in $\mathbf{1 3 b}$ since the two NH concerned are involved in amide links. Hence the activity of this compound probably depends on other factors e.g. $\pi-\pi$ stacking effects between the $3,3^{\prime}, 10,10^{\prime}$-phenyl substituents of the molecule and aromatic rings of Phe, Tyr and (or) Trp units of the transmembrane.

\section{Conclusion}

Variously substituted bis-diketal aminolactams and bis-diketal diamines were prepared in two and three steps respectively from previously and rapidly synthesized macrocyclic diketal dilactams [11a]. The first step, which corresponded to the formation of singly reduced compounds 4-6, was efficient in a 3$14 \times 10^{-3} \mathrm{M}$ concentration range for five of the six series, but failed from the cis methyl dilactam $2 \mathrm{c}$, the reduction of which led mainly to diamine 8c, irrespective of the concentration. After a coupling stage which gave bis-diketal aminolactams 13-15 in 37-72\% yield, the final reduction step allowed the isolation of bis-diketal diamines $18 b(R=M e)$ and $19 c(R=H)$, while a partial reaction or a major benzyl cleavage accompanied the reduction of the phenyl product $\mathbf{1 3 b}$.

Biological assays made on the unreduced and reduced dimer macrocycles showed that the bis-diphenyl diketal aminolactam 13b was the most promising compound, with both weak anti-HIV and antiproliferative activities. These results lead to consider a mode of action different from that of AMD 3100, and indicate the probable importance of phenyl groups susceptible to induce $\pi-\pi$ stacking effects with aromatic units of the CXCR4 receptor. The development and biological evaluation of new analogs incorporating phenyl substituents would enable us to test this hypothesis.

\section{Experimental}

\subsection{Chemistry}

\subsubsection{General remarks}

Solvents were obtained dried as follows: tetrahydrofuran (THF) was distilled from benzophenone ketyl, $\mathrm{CH}_{2} \mathrm{Cl}_{2}$ and $\mathrm{CH}_{3} \mathrm{CN}$ were refluxed over and distilled from $\mathrm{CaH}_{2}, \mathrm{CH}_{3} \mathrm{OH}$ was distilled from magnesium. The organic layers were dried with $\mathrm{Mg}_{2} \mathrm{SO}_{4}$. Reactions were monitored by thin layer chromatography (TLC) on aluminium plates precoated with silica gel (Merck $60 \mathrm{~F}_{254}$ ) and spots visualized by UV light or by spraying with a ceric sulphate and ammonium molybdate acid solution. Flash chromatography was carried out with silica gel (Merck $0.040-0.063 \mathrm{~mm}$ ). Optical rotations were measured at the sodium D line $(589 \mathrm{~nm})$ using a $1 \mathrm{dm}$ quartz cell with a JASCO DIP-370 apparatus. IR spectra were scanned with a Perkin-Elmer 881 spectrophotometer $\left(v_{\max }\right.$ in $\left.\mathrm{cm}^{-1}\right)$. 1D $\left({ }^{1} \mathrm{H}\right.$ and ${ }^{13} \mathrm{C}-\mathrm{J}$ MOD) and 2D (COSY ${ }^{1} \mathrm{H}-{ }^{1} \mathrm{H}$, HSQC ${ }^{1} \mathrm{H}^{-13} \mathrm{C}$ ) NMR spectra were recorded with a Bruker Avance 400 spectrometer equipped with a ${ }^{1} \mathrm{H} /{ }^{13} \mathrm{C}$ probe $\left({ }^{1} \mathrm{H}: 400 \mathrm{MHz} ;{ }^{13} \mathrm{C}: 100 \mathrm{MHz}\right)$, and $2 \mathrm{D} \mathrm{HMBC}{ }^{1} \mathrm{H}-{ }^{13} \mathrm{C}$ NMR spectra with an Avance 500 apparatus long dist $7.7 \mathrm{~Hz}\left({ }^{1} \mathrm{H}: 500 \mathrm{MHz} ;{ }^{13} \mathrm{C}: 125 \mathrm{MHz}\right)$. Chemical shifts $\delta$ were noted in parts per million (ppm) and splitting patterns designated as follows: singlet (s), doublet (d), triplet (t), quartet (q), doubled doublet (dd), doublet of doublet of doublet (ddd), doubled triplet (dt), multiplet (m), broad signal (br. s). The solvent $\left(\mathrm{CDCl}_{3}\right)$ was taken as internal reference $\left[\delta=7.27 \mathrm{ppm}\right.$ for ${ }^{1} \mathrm{H} \mathrm{NMR}$ and $77.1 \mathrm{ppm}$ (central line) for $\left.{ }^{13} \mathrm{C} \mathrm{NMR}\right]$. Proton and carbon atoms were assigned according to the numbering indicated in Fig. 2 and Scheme 1. Mass spectra were recorded with either an HP 5989B (CI) or a micro Q-TOF Waters (ESI) apparatus. 
To a suspension of $\mathrm{LiAlH}_{4}$ (10 equiv.) in dry THF $\left(0.5 \mathrm{~mL}\right.$ for $1 \mathrm{mmol}$ of LAH) was added $\mathrm{NEt}_{3}(0.01-$ 0.04 equiv./LAH), and dropwise a solution of diketal dilactam ( 1 equiv.) in dry THF $\left(c_{\text {substrate }}=3-\right.$ $\left.14 \times 10^{-3} \mathrm{M}\right)$. The reaction mixture was heated at reflux while stirring for 9-18 h. Excess hydride was destroyed by adding $\mathrm{H}_{2} \mathrm{O}$ (10 equiv./LAH). The precipitates were filtered and washed with THF and $\mathrm{CH}_{2} \mathrm{Cl}_{2}$. The organic layer was dried with $\mathrm{MgSO}_{4}$ and evaporated in vacuo. Purification by flash chromatography (silicagel, $\mathrm{Et}_{2} \mathrm{O} / \mathrm{MeOH}$ ) yielded diketal aminolactams and diketal diamines.

6.1.2.1. (3R,7R,10R,14S)-Diphenyl diketal aminolactam $(4 \mathrm{~b}),(3 R, 7 R, 10 R, 14 S)$-diphenyl diketal diamine (7b)

The preparation of these compounds was described previously [12b].

6.1.2.2. (3R,7S,10R,14S)-Diphenyl diketal aminolactam $(4 \mathrm{c}),(3 R, 7 S, 10 R, 14 S)$-Diphenyl diketal diamine (7c), and $(3 R, 10 R, 14 S)$-diphenyl unsaturated ketal aminolactam (10)

These compounds were prepared from diphenyl diketal dilactam 1c $(66.4 \mathrm{mg}, 0.15 \mathrm{mmol}), \mathrm{LiAlH}_{4}$ (57 mg, 1.5 mmol, 10 equiv.), $\mathrm{NEt}_{3}$ (1 M solution in THF, $30 \mu \mathrm{L}, 30 \mu \mathrm{mol}, 0.02$ equiv./LAH), and THF $\left(19 \mathrm{~mL}, c_{\text {substrat }}=8.0 \times 10^{-3} \mathrm{M}\right)$ according to the general procedure (reaction time $\left.9 \mathrm{~h}\right)$. Excess hydride was destroyed by adding $\mathrm{H}_{2} \mathrm{O}(270 \mu \mathrm{L}, 15 \mathrm{mmol})$. Chromatography ( $\mathrm{Et}_{2} \mathrm{O} / \mathrm{MeOH}$, gradient 100:0-60:40) afforded diketal aminolactam $4 \mathbf{c}(18.9 \mathrm{mg}, 44 \mu \mathrm{mol}, 35 \%)$, diketal diamine 7c $(5.2 \mathrm{mg}, 12.6 \mu \mathrm{mol}, 10 \%)$ and unsaturated ketal aminolactam $10(4.0 \mathrm{mg}, 10.1 \mu \mathrm{mol}, 8 \%)$ with $84 \%$ conversion rate. Data of $\mathbf{4 c}, \mathbf{7 c}$, and 10 were given previously [12a].

6.1.2.3. (3S,7S,10S,14R)-Dimethyl diketal aminolactam (5b), (3S,7S,10S,14R)-dimethyl diketal diamine (8b), and $(3 S, 10 S, 14 R)$-dimethyl unsaturated ketal aminolactam (11)

These compounds were prepared from methyl diketal dilactam $\mathbf{2 b}(63.7 \mathrm{mg}, 0.20 \mathrm{mmol}), \mathrm{LiAlH}_{4}(76 \mathrm{mg}$, $2.0 \mathrm{mmol}, 10$ equiv.), $\mathrm{NEt}_{3}$ ( $1 \mathrm{M}$ solution in THF, $20 \mu \mathrm{L}, 20 \mu \mathrm{mol}, 0.01$ equiv./LAH), and THF $(25 \mathrm{~mL}$, $c_{\text {substrat }}=8.0 \times 10^{-3} \mathrm{M}$ ) according to the general procedure (reaction time $18 \mathrm{~h}$ ). Excess hydride was destroyed by adding $\mathrm{H}_{2} \mathrm{O}(360 \mu \mathrm{L}, 20 \mathrm{mmol})$. Chromatography $\left(\mathrm{Et}_{2} \mathrm{O} / \mathrm{MeOH}\right.$, gradient 100:0-0:100) afforded diketal aminolactam $5 \mathbf{b}(15.0 \mathrm{mg}, 49.3 \mu \mathrm{mol}, 28 \%)$, diketal diamine $\mathbf{8 b}(6.1 \mathrm{mg}, 21.1 \mu \mathrm{mol}$, $12 \%)$ and unsaturated ketal aminolactam $11(0.9 \mathrm{mg}, 3.5 \mu \mathrm{mol}, 2 \%)$ with $88 \%$ conversion rate. Data of $\mathbf{5 b}, \mathbf{8 b}$, and $\mathbf{1 1}$ were given previously [12b].

6.1.2.4. (3S,7R,10S,14R)-Dimethyl diketal aminolactam $(5 \mathrm{c}),(3 S, 7 R, 10 S, 14 R)$-dimethyl diketal diamine $(8 \mathrm{c})$ and $(3 S, 10 S, 14 R)$-dimethyl unsaturated ketal aminolactam (11)

These compounds were prepared from methyl diketal dilactam $2 \mathbf{c}(63.7 \mathrm{mg}, 0.20 \mathrm{mmol}), \mathrm{LiAlH}_{4}$ (45.5 mg, 1.2 mmol, 6 equiv.), $\mathrm{NEt}_{3}$ ( $1 \mathrm{M}$ solution in THF, $12 \mu \mathrm{L}, 12 \mu \mathrm{mol}, 0.01$ equiv./LAH), and THF $\left(50 \mathrm{~mL}, c_{\text {substrat }}=4.0 \times 10^{-3} \mathrm{M}\right)$ according to the general procedure (reaction time $\left.9 \mathrm{~h}\right)$. Excess hydride was destroyed by adding $\mathrm{H}_{2} \mathrm{O}(216 \mu \mathrm{L}, 12 \mathrm{mmol})$. Chromatography $\left(\mathrm{Et}_{2} \mathrm{O} / \mathrm{MeOH}\right.$, gradient 100:0-0:100) afforded diketal aminolactam 5c (4.4 mg, $14.4 \mu \mathrm{mol}, 12 \%)$, diketal diamine 8c (7.7 mg, $26.4 \mu \mathrm{mol}, 22 \%)$ and unsaturated ketal aminolactam $11(0.3 \mathrm{mg}, 1.2 \mu \mathrm{mol}, 1 \%)$ with $60 \%$ conversion rate. Data of $\mathbf{5 c}, \mathbf{8 c}$, and 11 were given previously [12b].

6.1.2.5. $\left(7 S^{*}, 14 R^{*}\right)$-Diketal aminolactam $(6 \mathrm{~b}),\left(7 S^{*}, 14 R^{*}\right)$-diketal diamine $(9 \mathrm{~b})$, and $14 R^{*}$-unsaturated ketal aminolactam (12)

These compounds were prepared from diketal dilactam $\mathbf{3 b}(72.6 \mathrm{mg}, 0.25 \mathrm{mmol}), \mathrm{LiAlH}_{4}(95 \mathrm{mg}$, $2.5 \mathrm{mmol}, 10$ equiv.), $\mathrm{NEt}_{3}$ ( $1 \mathrm{M}$ solution in THF, $50 \mu \mathrm{L}, 50 \mu \mathrm{mol}, 0.02$ equiv./LAH), and THF (50 mL, $c_{\text {substrat }}=5.0 \times 10^{-3} \mathrm{M}$ ) according to the general procedure (reaction time $9 \mathrm{~h}$ ). Excess hydride was destroyed by adding $\mathrm{H}_{2} \mathrm{O}(450 \mu \mathrm{L}, 25 \mathrm{mmol})$. Chromatography $\left(\mathrm{Et}_{2} \mathrm{O} / \mathrm{MeOH}\right.$, gradient 100:0-0:100) afforded diketal aminolactam $\mathbf{6 b}(14.5 \mathrm{mg}, 52.6 \mu \mathrm{mol}, 27 \%)$, diketal diamine 9b $(8.2 \mathrm{mg}, 31.2 \mu \mathrm{mol}$, $16 \%)$ and unsaturated ketal aminolactam $12(1.0 \mathrm{mg}, 4.0 \mu \mathrm{mol}, 2 \%)$ with $78 \%$ conversion rate.

Data for 6b: $R_{\mathrm{f}}=0.50\left(\mathrm{MeOH} / \mathrm{NH}_{4} \mathrm{OH}: 99.5 / 0.5,2\right.$ elutions). IR $\left(\mathrm{CHCl}_{3}\right): v_{\max } 3450,3420,3340(\mathrm{NH})$; 1660 (CO). ${ }^{1} \mathrm{H}$ NMR and COSY ${ }^{1} \mathrm{H}-{ }^{1} \mathrm{H}\left(400 \mathrm{MHz}, \mathrm{CDCl}_{3}\right): \delta_{\mathrm{H}} 1.89$ (dddd, $1 \mathrm{H}, \mathrm{H}-13 \mathrm{~B},{ }^{2} J=14.5 \mathrm{~Hz}$, ${ }^{3} J=7.6,2.7,2.4 \mathrm{~Hz}$ ), 1.99 (dddd, $1 \mathrm{H}, \mathrm{H}-13 \mathrm{~A},{ }^{2} J=14.5 \mathrm{~Hz},{ }^{3} J=8.7,8.1,2.8 \mathrm{~Hz}$ ), 2.53 (dd, 1H, H-6B, ${ }^{2} J=14.6 \mathrm{~Hz},{ }^{3} J=1.9 \mathrm{~Hz}$ ), 2.56 (br. s, $1 \mathrm{H}, \mathrm{NH}$ amine), 2.70 (dd, $1 \mathrm{H}, \mathrm{H}-6 \mathrm{~A},{ }^{2} J=14.6 \mathrm{~Hz},{ }^{3} \mathrm{~J}=8.6 \mathrm{~Hz}$ ), 
2.75 (ddd, 1H, H-12B, ${ }^{2} J=11.5 \mathrm{~Hz},{ }^{3} J=8.7,2.7 \mathrm{~Hz}$ ), 2.81 (ddd, $1 \mathrm{H}, \mathrm{H}-10 \mathrm{~B},{ }^{2} J=12.6 \mathrm{~Hz},{ }^{3} J=5.3$, $2.6 \mathrm{~Hz}$ ), 2.89 (ddd, $1 \mathrm{H}, \mathrm{H}-12 \mathrm{~A},{ }^{2} J=11.5 \mathrm{~Hz},{ }^{3} \mathrm{~J}=7.6,2.8 \mathrm{~Hz}$ ), 2.91 (ddd, $1 \mathrm{H}, \mathrm{H}-10 \mathrm{~A},{ }^{2} J=12.6 \mathrm{~Hz}$, $\left.{ }^{3} J=8.5,2.8 \mathrm{~Hz}\right), 3.33\left(\mathrm{~s}, 3 \mathrm{H}, \mathrm{OCH}_{3}\right), 3.36\left(\mathrm{~s}, 3 \mathrm{H}, \mathrm{OCH}_{3}\right), 3.38\left(\mathrm{dddd}, 1 \mathrm{H}, \mathrm{H}-3 \mathrm{~B},{ }^{2} J=13.7,{ }^{3} J=6.4,5.2\right.$, $2.4 \mathrm{~Hz}$ ), 3.51 (ddd, $1 \mathrm{H}, \mathrm{H}-2 \mathrm{~B},{ }^{2} J=9.9 \mathrm{~Hz},{ }^{3} J=7.9,2.4 \mathrm{~Hz}$ ), 3.55 (ddd, $1 \mathrm{H}, \mathrm{H}-9 \mathrm{~B},{ }^{2} J=9.9 \mathrm{~Hz},{ }^{3} J=8.5$, $2.6 \mathrm{~Hz}$ ), 3.63 (dddd, $1 \mathrm{H}, \mathrm{H}-3 \mathrm{~A},{ }^{2} J=13.7 \mathrm{~Hz},{ }^{3} J=7.9,5.6,2.6 \mathrm{~Hz}$ ), 3.82 (ddd, $1 \mathrm{H}, \mathrm{H}-2 \mathrm{~A},{ }^{2} J=9.9 \mathrm{~Hz}$, $\left.{ }^{3} J=6.4,2.6 \mathrm{~Hz}\right), 3.96\left(\mathrm{ddd}, 1 \mathrm{H}, \mathrm{H}-9 \mathrm{~A},{ }^{2} J=9.9 \mathrm{~Hz},{ }^{3} J=5.3,2.8 \mathrm{~Hz}\right), 4.62\left(\mathrm{dd}, 1 \mathrm{H}, \mathrm{H}-14,{ }^{2} J=8.1\right.$, $2.4 \mathrm{~Hz}), 4.80\left(\mathrm{dd}, 1 \mathrm{H}, \mathrm{H}-7,{ }^{3} \mathrm{~J}=8.6,1.9 \mathrm{~Hz}\right), 7.00$ (br. s, $1 \mathrm{H}, \mathrm{NH}$ lactam). ${ }^{13} \mathrm{C}$ NMR and HSQC $\left(100 \mathrm{MHz}, \mathrm{CDCl}_{3}\right): \delta_{\mathrm{C}} 31.6(\mathrm{C}-13), 39.3(\mathrm{C}-3), 41.1(\mathrm{C}-6), 45.7(\mathrm{C}-12), 49.3(\mathrm{C}-10), 53.1\left(\mathrm{OCH}_{3}\right), 53.6$ $\left(\mathrm{OCH}_{3}\right), 64.1$ (C-2), 65.3 (C-9), 101.2 (C-7), 104.0 (C-14), 169.0 (CO). MS (ESI ): $\mathrm{m} / z(\%) 299(28)$ $[\mathrm{M}+\mathrm{Na}]^{+}, 277(100)[\mathrm{M}+\mathrm{H}]^{+}, 245(87)\left[\mathrm{M}+\mathrm{H}-\mathrm{CH}_{3} \mathrm{OH}\right]^{+}$. HRMS (ESI $)$: calcd. for $\mathrm{C}_{12} \mathrm{H}_{25} \mathrm{~N}_{2} \mathrm{O}_{5}$ $[\mathrm{M}+\mathrm{H}]^{+} 277.1763$, found 277.1767 .

Data of $9 \mathbf{b}$ were given previously [12b].

Data for 12: $R_{\mathrm{f}}=0.60\left(\mathrm{MeOH}, 3\right.$ elutions). IR $\left(\mathrm{CHCl}_{3}\right): v_{\max } 3429(\mathrm{NH}), 1663(\mathrm{CO}), 1604(\mathrm{C}=\mathrm{C}) .{ }^{1} \mathrm{H}$ NMR and COSY ${ }^{1} \mathrm{H}^{1}{ }^{1} \mathrm{H}\left(400 \mathrm{MHz}, \mathrm{CDCl}_{3}\right): \delta_{\mathrm{H}} 1.60$ (br. s, $1 \mathrm{H}, \mathrm{NH}$ amine), 1.82 (dddd, $1 \mathrm{H}, \mathrm{H}-13 \mathrm{~B}$, ${ }^{2} J=14.3 \mathrm{~Hz},{ }^{3} J=7.2,2.2,2.1 \mathrm{~Hz}$ ), 1.99 (dddd, $1 \mathrm{H}, \mathrm{H}-13 \mathrm{~A},{ }^{2} J=14.3 \mathrm{~Hz},{ }^{3} J=9.3,8.5,2.3 \mathrm{~Hz}$ ), 2.70 (ddd, 1H, H-12B, ${ }^{2} J=11.5,{ }^{3} J=9.3,2.1 \mathrm{~Hz}$ ), 2.88 (m, 3H, H-10A, H-10B, H-12A), 3.28 (dddd, 1H, H$\left.3 \mathrm{~B},{ }^{2} J=11.0,{ }^{3} J=9.0,3.5,2.5 \mathrm{~Hz}\right), 3.33\left(\mathrm{~s}, 3 \mathrm{H}, \mathrm{OCH}_{3}\right), 3.47\left(\mathrm{td}, 1 \mathrm{H}, \mathrm{H}-2 \mathrm{~B},{ }^{2} J=10.0,{ }^{3} \mathrm{~J}=2.5 \mathrm{~Hz}\right), 3.85$ (ddt, $\left.1 \mathrm{H}, \mathrm{H}-3 \mathrm{~A},{ }^{2} J=11.0,{ }^{3} J=10.0,3.5 \mathrm{~Hz}\right), 3.91\left(\mathrm{dt}, 1 \mathrm{H}, \mathrm{H}-2 \mathrm{~A},{ }^{2} J=10.0,{ }^{3} J=3.5 \mathrm{~Hz}\right), 4.07$ (ddd, $1 \mathrm{H}$, $\mathrm{H}-9 \mathrm{~B},{ }^{2} J=10.3,{ }^{3} J=4.3,4.1 \mathrm{~Hz}$ ), 4.21 (ddd, $1 \mathrm{H}, \mathrm{H}-9 \mathrm{~A},{ }^{2} J=10.3,{ }^{3} J=5.9,4.9 \mathrm{~Hz}$ ), 4.58 (dd, 1H, H-14, $\left.{ }^{3} J=8.5,2.2 \mathrm{~Hz}\right), 5.01\left(\mathrm{~d}, 1 \mathrm{H}, \mathrm{H}-6,{ }^{3} J=6.9 \mathrm{~Hz}\right), 6.48\left(\mathrm{~d}, 1 \mathrm{H}, \mathrm{H}-7,{ }^{3} J=6.9 \mathrm{~Hz}\right), 7.60$ (br. s, $1 \mathrm{H}, \mathrm{NH}$ lactam). MS (ESI $\left.{ }^{+}\right): m / z(\%) 267(100)[\mathrm{M}+\mathrm{Na}]^{+}, 245(52)[\mathrm{M}+\mathrm{H}]^{+}, 213$ (98) $\left[\mathrm{M}+\mathrm{H}-\mathrm{CH}_{3} \mathrm{OH}\right]^{+}$. HRMS (ESI ${ }^{+}$): calcd. for $\mathrm{C}_{11} \mathrm{H}_{21} \mathrm{~N}_{2} \mathrm{O}_{4}[\mathrm{M}+\mathrm{H}]^{+} 245.1501$, found 245.1514 .

\subsubsection{6. $\left(7 R^{*}, 14 R^{*}\right)$-Diketal aminolactam $(6 \mathrm{c})$ and $\left(7 R^{*}, 14 R^{*}\right)$-diketal diamine $(9 \mathrm{c})$}

These compounds were prepared from methyl diketal dilactam 3c (116.1 mg, $0.40 \mathrm{mmol}), \mathrm{LiAlH}_{4}$ (152 mg, 4.0 mmol, 10 equiv.), $\mathrm{NEt}_{3}$ (1 M solution in THF, $80 \mu \mathrm{L}, 80 \mu \mathrm{mol}, 0.02$ equiv./LAH), and THF $\left(133 \mathrm{~mL}, c_{\text {substrate }}=3.0 \times 10^{-3} \mathrm{M}\right)$ according to the general procedure (reaction time $\left.10 \mathrm{~h}\right)$. Excess hydride was destroyed by adding $\mathrm{H}_{2} \mathrm{O}(720 \mu \mathrm{L}, 40 \mathrm{mmol})$. Chromatography $\left(\mathrm{Et}_{2} \mathrm{O} / \mathrm{MeOH}\right.$, gradient 100:0-0:100) afforded diketal aminolactam $\mathbf{6 c}(22.5 \mathrm{mg}, 81.4 \mu \mathrm{mol}, 29 \%)$ and diketal diamine 9c $(10.3 \mathrm{mg}, 39.3 \mu \mathrm{mol}$, $14 \%$ ) with $70 \%$ conversion rate. Data of $\mathbf{6 c}$ and $9 \mathbf{c}$ were given previously [12b].

\subsubsection{Bis-diketal aminolactams: general procedure}

A suspension of diketal aminolactam, $\alpha, \alpha^{\prime}$-dibromo- $p$-xylene $\left(0.5-0.6\right.$ equiv.) and $\mathrm{K}_{2} \mathrm{CO}_{3}(1.0-1.2$ equiv.) in dry $\mathrm{CH}_{3} \mathrm{CN}\left(c_{\text {substrate }}=50 \times 10^{-3} \mathrm{M}\right)$ was heated at reflux while stirring for 8-9 h. After evaporation of the solvent, the residue was dissolved in $\mathrm{CH}_{2} \mathrm{Cl}_{2}$. The precipitate was filtered and washed with $\mathrm{CH}_{2} \mathrm{Cl}_{2}$. The organic layer was dried with $\mathrm{Mg}_{2} \mathrm{SO}_{4}$ and evaporated in vacuo. Purification by flash chromatography (silica gel, $\mathrm{Et}_{2} \mathrm{O} / \mathrm{MeOH}$ ) yielded bis-diketal aminolactams and small amounts of bromo diketal aminolactams.

6.1.3.1. Bis-(3R,7R,10R,14S)-diphenyl diketal aminolactam $(13 \mathrm{~b})$ and $(3 R, 7 R, 10 R, 14 S)$-bromoxylyl diphenyl diketal aminolactam (16b)

These compounds were prepared from diphenyl diketal aminolactam $4 \mathbf{b}(25.7 \mathrm{mg}, 60.0 \mu \mathrm{mol}), \alpha, \alpha^{\prime}-$ dibromo- $p$-xylene (7.9 mg, $30.0 \mu \mathrm{mol}, 0.5$ equiv.), $\mathrm{K}_{2} \mathrm{CO}_{3}\left(8.3 \mathrm{mg}, 60.0 \mu \mathrm{mol}, 1.0\right.$ equiv.), and $\mathrm{CH}_{3} \mathrm{CN}$ $\left(1.2 \mathrm{~mL}, c_{\text {substrate }}=50 \times 10^{-3} \mathrm{M}\right)$ according to the general procedure (reaction time $\left.8 \mathrm{~h}\right)$. Chromatography ( $\mathrm{Et}_{2} \mathrm{O} / \mathrm{MeOH}$, gradient: 100:0-50:50) afforded bis-diketal aminolactam 13b (16.0 mg, $\left.16.8 \mu \mathrm{mol}, 61 \%\right)$ and bromoxylyl diphenyl diketal aminolactam $\mathbf{1 6 b}(5.4 \mathrm{mg}, 8.8 \mu \mathrm{mol}, 16 \%)$ with $92 \%$ conversion rate. Data for 13b: $R_{\mathrm{f}}=0.40\left(\mathrm{Et}_{2} \mathrm{O} / \mathrm{MeOH}, 98: 2,3\right.$ elutions $)$. [ $\left.\alpha\right]_{\mathrm{D}}{ }^{25}-68.0\left(c 0.90, \mathrm{CHCl}_{3}\right)$. IR $\left(\mathrm{CHCl}_{3}\right): v_{\max }$ $3367(\mathrm{NH}), 1665(\mathrm{CO}) .{ }^{1} \mathrm{H}$ NMR and COSY ${ }^{1} \mathrm{H}-{ }^{1} \mathrm{H}\left(400 \mathrm{MHz}, \mathrm{CDCl}_{3}\right): \delta_{\mathrm{H}} 1.86(\mathrm{~m}, 4 \mathrm{H}, 2 \mathrm{H}-13 \mathrm{~A}, 2 \mathrm{H}-$ 13B), $2.56\left(\mathrm{dd}, 2 \mathrm{H}, 2 \mathrm{H}-6 \mathrm{~B},{ }^{2} J=16.1 \mathrm{~Hz},{ }^{3} \mathrm{~J}=1.5 \mathrm{~Hz}\right), 2.61\left(\mathrm{dt}, 2 \mathrm{H}, 2 \mathrm{H}-12 \mathrm{~B},{ }^{2} \mathrm{~J}=12.8 \mathrm{~Hz},{ }^{3} \mathrm{~J}=4.9 \mathrm{~Hz}\right)$, $2.84\left(\mathrm{dd}, 2 \mathrm{H}, 2 \mathrm{H}-6 \mathrm{~A},{ }^{2} \mathrm{~J}=16.1 \mathrm{~Hz},{ }^{3} \mathrm{~J}=8.7 \mathrm{~Hz}\right), 3.10\left(\mathrm{~s}, 6 \mathrm{H}, 2 \mathrm{OCH}_{3}\right), 3.16\left(\mathrm{~s}, 6 \mathrm{H}, 2 \mathrm{OCH}_{3}\right), 3.30(\mathrm{~d}, 2 \mathrm{H}$, $\left.2 \mathrm{H}-15 \mathrm{~B},{ }^{2} J=14.7 \mathrm{~Hz}\right), 3.34(\mathrm{~m}, 2 \mathrm{H}, 2 \mathrm{H}-12 \mathrm{~A}), 3.76\left(\mathrm{dd}, 2 \mathrm{H}, 2 \mathrm{H}-2 \mathrm{~B},{ }^{2} J=9.8 \mathrm{~Hz},{ }^{3} \mathrm{~J}=3.2 \mathrm{~Hz}\right), 3.80(\mathrm{~d}$, $\left.2 \mathrm{H}, 2 \mathrm{H}-15 \mathrm{~A},{ }^{2} J=14.7 \mathrm{~Hz}\right), 3.92\left(\mathrm{dd}, 2 \mathrm{H}, 2 \mathrm{H}-9 \mathrm{~B},{ }^{2} J=10.0 \mathrm{~Hz},{ }^{3} J=2.2 \mathrm{~Hz}\right), 3.97(\mathrm{dd}, 2 \mathrm{H}, 2 \mathrm{H}-10$, $\left.{ }^{3} J=7.3,2.2 \mathrm{~Hz}\right), 4.10\left(\mathrm{dd}, 2 \mathrm{H}, 2 \mathrm{H}-2 \mathrm{~A},{ }^{2} J=9.8 \mathrm{~Hz},{ }^{3} J=2.9 \mathrm{~Hz}\right), 4.11\left(\mathrm{dd}, 2 \mathrm{H}, 2 \mathrm{H}-9 \mathrm{~A},{ }^{2} J=10.0 \mathrm{~Hz}\right.$, 
$\left.{ }^{3} J=7.3 \mathrm{~Hz}\right), 4.79\left(\mathrm{dd}, 2 \mathrm{H}, 2 \mathrm{H}-7,{ }^{3} J=8.7,1.5 \mathrm{~Hz}\right), 4.84\left(\mathrm{t}, 2 \mathrm{H}, 2 \mathrm{H}-14,{ }^{3} J=5.3 \mathrm{~Hz}\right), 5.28$ (ddd, 2H, 2H-3, $\left.{ }^{3} J=7.9,3.2,2.9 \mathrm{~Hz}\right), 7.28-7.42(\mathrm{~m}, 24 \mathrm{H}, 24 \mathrm{Ar}-\mathrm{H}), 7.90\left(\mathrm{~d}, 2 \mathrm{H}, 2 \mathrm{NH}\right.$ lactam, $\left.{ }^{3} J=7.9 \mathrm{~Hz}\right) .{ }^{13} \mathrm{C}$ NMR and HSQC (100 MHz, $\left.\mathrm{CDCl}_{3}\right): \delta_{\mathrm{C}} 31.1(2 \mathrm{C}-13), 40.0(2 \mathrm{C}-6), 44.7(2 \mathrm{C}-12), 52.0\left(2 \mathrm{OCH}_{3}\right), 52.3(2 \mathrm{C}-3)$, $53.4\left(2 \mathrm{CH}_{3}\right), 53.5(2 \mathrm{C}-15), 63.4$ (2C-10), 66.4 (2C-9), 70.5 (2C-2), 100.1 (2C-7), 102.3 (2C-14), 126.7129.4 (14 ArCH), 136.6 (2 ArC), 138.3 (2 ArC), 140.4 (2 ArC), 168.3 (2CO). MS (ESI $)^{\dagger}: m / z(\%) 981$ (21) $[\mathrm{M}+\mathrm{Na}]^{+}, 959(7)[\mathrm{M}+\mathrm{H}]^{+}, 451(100)\left[\mathrm{C}_{24} \mathrm{H}_{32} \mathrm{~N}_{2} \mathrm{O}_{5}+\mathrm{Na}\right]^{+}, 429(84)\left[\mathrm{C}_{24} \mathrm{H}_{32} \mathrm{~N}_{2} \mathrm{O}_{5}+\mathrm{H}\right]^{+}, 339$ (42). HRMS (ESI ${ }^{+}$): calcd. for $\mathrm{C}_{56} \mathrm{H}_{71} \mathrm{~N}_{4} \mathrm{O}_{10}[\mathrm{M}+\mathrm{H}]^{+}$959.5170, found 959.5210 .

Data for 16b: $R_{\mathrm{f}}=0.60\left(\mathrm{Et}_{2} \mathrm{O}\right) .[\alpha]_{\mathrm{D}}{ }^{25}-56.6\left(c 0.57, \mathrm{CHCl}_{3}\right)$. IR $\left(\mathrm{CHCl}_{3}\right) v_{\max } 3360(\mathrm{NH}), 1660(\mathrm{CO}) .{ }^{1} \mathrm{H}$ NMR and COSY ${ }^{1} \mathrm{H}^{1}{ }^{\mathrm{H}}\left(400 \mathrm{MHz}, \mathrm{CDCl}_{3}\right): \delta_{\mathrm{H}} 1.85(\mathrm{~m}, 2 \mathrm{H}, \mathrm{H}-13 \mathrm{~A}, \mathrm{H}-13 \mathrm{~B}), 2.55$ (ddd, 1H, H-12B, $\left.{ }^{2} J=11.8 \mathrm{~Hz},{ }^{3} J=4.7,0.5 \mathrm{~Hz}\right), 2.57\left(\mathrm{dd}, 1 \mathrm{H}, \mathrm{H}-6 \mathrm{~B},{ }^{2} J=16.2 \mathrm{~Hz},{ }^{3} J=1.6 \mathrm{~Hz}\right), 2.83(\mathrm{dd}, 1 \mathrm{H}, \mathrm{H}-6 \mathrm{~A}$, $\left.{ }^{2} J=16.2 \mathrm{~Hz},{ }^{3} J=8.7 \mathrm{~Hz}\right), 3.11\left(\mathrm{~s}, 3 \mathrm{H}, 7-\mathrm{OCH}_{3}\right), 3.14\left(\mathrm{~s}, 3 \mathrm{H}, 14-\mathrm{OCH}_{3}\right), 3.31(\mathrm{~d}, 1 \mathrm{H}, \mathrm{H}-15 \mathrm{~B}$, ${ }^{2} J=15.0 \mathrm{~Hz}$ ), 3.32 (ddd, $\left.1 \mathrm{H}, \mathrm{H}-12 \mathrm{~A},{ }^{2} J=11.8 \mathrm{~Hz},{ }^{3} J=10.2,6.5 \mathrm{~Hz}\right), 3.74\left(\mathrm{dd}, 1 \mathrm{H}, \mathrm{H}-2 \mathrm{~B},{ }^{2} J=10.0 \mathrm{~Hz}\right.$, $\left.{ }^{3} J=3.3 \mathrm{~Hz}\right), 3.79$ (d, $\left.1 \mathrm{H}, \mathrm{H}-15 \mathrm{~A},{ }^{2} J=15.0 \mathrm{~Hz}\right), 3.89\left(\mathrm{dd}, 1 \mathrm{H}, \mathrm{H}-9 \mathrm{~B},{ }^{2} J=10.2 \mathrm{~Hz},{ }^{3} J=2.6 \mathrm{~Hz}\right), 3.96(\mathrm{dd}$, $\left.1 \mathrm{H}, \mathrm{H}-10,{ }^{3} J=8.4,2.6 \mathrm{~Hz}\right), 4.09\left(\mathrm{dd}, 1 \mathrm{H}, \mathrm{H}-2 \mathrm{~A},{ }^{2} J=10.0 \mathrm{~Hz},{ }^{3} J=2.7 \mathrm{~Hz}\right), 4.13(\mathrm{dd}, 1 \mathrm{H}, \mathrm{H}-9 \mathrm{~A}$, $\left.{ }^{2} J=10.2 \mathrm{~Hz},{ }^{3} J=8.4 \mathrm{~Hz}\right), 4.52\left(\mathrm{~s}, 2 \mathrm{H}, \mathrm{CH}_{2} \mathrm{Br}\right), 4.78\left(\mathrm{dd}, 1 \mathrm{H}, \mathrm{H}-14,{ }^{3} J=5.9,5.3 \mathrm{~Hz}\right), 4.79$ (dd, 1H, H-7, ${ }^{3} J=8.7,1.6 \mathrm{~Hz}$ ), 5.27 (ddd, $\left.1 \mathrm{H}, \mathrm{H}-3,{ }^{3} \mathrm{~J}=7.6,3.3,2.7 \mathrm{~Hz}\right), 7.22-7.42(\mathrm{~m}, 14 \mathrm{H}, 14 \mathrm{Ar}-\mathrm{H}), 7.90(\mathrm{~d}, 1 \mathrm{H}$, $\mathrm{NH}$ lactam, $\left.{ }^{3} \mathrm{~J}=7.6 \mathrm{~Hz}\right) .{ }^{13} \mathrm{C}$ NMR and HSQC $\left(100 \mathrm{MHz}, \mathrm{CDCl}_{3}\right): \delta_{\mathrm{C}} 31.1(\mathrm{C}-13), 33.6\left(\mathrm{CH}_{2} \mathrm{Br}\right), 40.1$ (C-6), $44.4(\mathrm{C}-12), 52.1\left(14-\mathrm{OCH}_{3}\right), 52.2(\mathrm{C}-3), 53.2\left(7-\mathrm{OCH}_{3}\right), 53.4(\mathrm{C}-15), 63.6(\mathrm{C}-10), 66.3(\mathrm{C}-9)$, 70.6 (C-2), 100.0 (C-7), 102.3 (C-14), 127.0-129.4 (14 Ar-CH), 136.1 (Ar-C), 136.6 (Ar-C), 140.2 (ArC), $140.4(\mathrm{Ar}-\mathrm{C}), 168.2(\mathrm{CO})$. MS $\left(\mathrm{ESI}^{+}\right): \mathrm{m} / z(\%) 635(9)\left[\mathrm{M}+\mathrm{Na}\left({ }^{81} \mathrm{Br}\right)\right]^{+}, 633(9)\left[\mathrm{M}+\mathrm{Na}\left({ }^{79} \mathrm{Br}\right)\right]^{+}$, $613(98)\left[\mathrm{M}+\mathrm{H}\left({ }^{81} \mathrm{Br}\right)\right]^{+}, 611(100)\left[\mathrm{M}+\mathrm{H}\left({ }^{79} \mathrm{Br}\right)\right]^{+}, 555$ (16), 553 (16). HRMS (ESI $\left.{ }^{+}\right)$: calcd. for $\mathrm{C}_{32} \mathrm{H}_{40}$ ${ }^{79} \mathrm{BrN}_{2} \mathrm{O}_{5}[\mathrm{M}+\mathrm{H}]^{+}$611.2121, found 611.2132.

6.1.3.2. Bis-(3R,7S,10R,14S)-diphenyl diketal aminolactam $(13 \mathrm{c})$ and $(3 R, 7 S, 10 R, 14 S)$-bromoxylyl diphenyl diketal aminolactam (16c)

These compounds were prepared from diphenyl diketal aminolactam 4c (17.1 mg, $40.0 \mu \mathrm{mol}), \alpha, \alpha^{\prime}-$ dibromo- $p$-xylene $\left(6.3 \mathrm{mg}, 24.0 \mu \mathrm{mol}, 0.6\right.$ equiv.), $\mathrm{K}_{2} \mathrm{CO}_{3}\left(6.6 \mathrm{mg}, 48.0 \mu \mathrm{mol}, 1.2\right.$ equiv.), and $\mathrm{CH}_{3} \mathrm{CN}$ $\left(0.8 \mathrm{~mL}, c_{\text {substrate }}=50 \times 10^{-3} \mathrm{M}\right)$ according to the general procedure (reaction time $\left.8 \mathrm{~h}\right)$. Chromatography $\left(\mathrm{Et}_{2} \mathrm{O} / \mathrm{MeOH}\right.$, gradient 100:0-50:50) afforded bis-diketal aminolactam 13c $(7.1 \mathrm{mg}, 7.4 \mu \mathrm{mol}, 37 \%)$ and bromoxylyl diphenyl diketal aminolactam 16c $(4.7 \mathrm{mg}, 7.7 \mu \mathrm{mol}, 19 \%)$ with $100 \%$ conversion rate.

Data for 13c: $R_{\mathrm{f}}=0.33\left(\mathrm{Et}_{2} \mathrm{O} / \mathrm{MeOH}, 98: 2\right)$. $[\alpha]_{\mathrm{D}}{ }^{25}-56.1\left(c 0.25 ; \mathrm{CHCl}_{3}\right)$. IR $\left(\mathrm{CHCl}_{3}\right): v_{\max } 3436$ and $3372(\mathrm{NH}), 1666(\mathrm{CO}) .{ }^{1} \mathrm{H}$ NMR and COSY ${ }^{1} \mathrm{H}-{ }^{1} \mathrm{H}\left(400 \mathrm{MHz}, \mathrm{CDCl}_{3}\right): \delta_{\mathrm{H}} 1.87(\mathrm{q}, 4 \mathrm{H}, 4 \mathrm{H}-13$, $\left.{ }^{3} J=6.4 \mathrm{~Hz}\right), 2.59\left(\mathrm{dd}, 2 \mathrm{H}, 2 \mathrm{H}-6 \mathrm{~B},{ }^{2} J=14.5 \mathrm{~Hz},{ }^{3} J=2.4 \mathrm{~Hz}\right), 2.63\left(\mathrm{dd}, 2 \mathrm{H}, 2 \mathrm{H}-6 \mathrm{~A},{ }^{2} J=14.5 \mathrm{~Hz}\right.$, $\left.{ }^{3} J=7.0 \mathrm{~Hz}\right), 2.76\left(\mathrm{dt}, 2 \mathrm{H}, 2 \mathrm{H}-12 \mathrm{~B},{ }^{2} J=12.9 \mathrm{~Hz},{ }^{3} J=6.4 \mathrm{~Hz}\right), 3.11\left(\mathrm{dt}, 2 \mathrm{H}, 2 \mathrm{H}-12 \mathrm{~A},{ }^{2} J=12.9 \mathrm{~Hz}\right.$, $\left.{ }^{3} J=6.4 \mathrm{~Hz}\right), 3.19\left(\mathrm{~s}, 12 \mathrm{H}, 4 \mathrm{OCH}_{3}\right), 3.43\left(\mathrm{~d}, 2 \mathrm{H}, 2 \mathrm{H}-15 \mathrm{~B},{ }^{2} J=14.3 \mathrm{~Hz}\right), 3.74(\mathrm{~d}, 2 \mathrm{H}, 2 \mathrm{H}-15 \mathrm{~A}$, $\left.{ }^{2} J=14.3 \mathrm{~Hz}\right), 3.83\left(\mathrm{dd}, 2 \mathrm{H}, 2 \mathrm{H}-9 \mathrm{~B},{ }^{2} J=10.3 \mathrm{~Hz},{ }^{3} J=3.3 \mathrm{~Hz}\right), 3.84\left(\mathrm{dd}, 2 \mathrm{H}, 2 \mathrm{H}-2 \mathrm{~B},{ }^{2} J=10.8 \mathrm{~Hz}\right.$, $\left.{ }^{3} J=4.2 \mathrm{~Hz},\right), 3.91\left(\mathrm{dd}, 2 \mathrm{H}, 2 \mathrm{H}-2 \mathrm{~A},{ }^{2} J=10.8 \mathrm{~Hz},{ }^{3} J=4.9 \mathrm{~Hz}\right), 3.92\left(\mathrm{dd}, 2 \mathrm{H}, 2 \mathrm{H}-10,{ }^{3} J=7.4,3.3 \mathrm{~Hz}\right)$, $4.23\left(\mathrm{dd}, 2 \mathrm{H}, 2 \mathrm{H}-9 \mathrm{~A},{ }^{2} J=10.3 \mathrm{~Hz},{ }^{3} J=7.4 \mathrm{~Hz}\right), 4.78\left(\mathrm{dd}, 2 \mathrm{H}, 2 \mathrm{H}-7,{ }^{3} J=7.0,2.4 \mathrm{~Hz}\right), 4.79(\mathrm{t}, 2 \mathrm{H}, 2 \mathrm{H}-$ $\left.14,{ }^{3} J=6.4 \mathrm{~Hz}\right), 5.24\left(\mathrm{ddd}, 2 \mathrm{H}, 2 \mathrm{H}-3,{ }^{3} J=7.7,4.9,4.2 \mathrm{~Hz}\right), 7.13\left(\mathrm{~d}, 2 \mathrm{H}, 2 \mathrm{NH}\right.$ lactam, $\left.{ }^{3} J=7.7 \mathrm{~Hz}\right)$, 7.28-7.40 (m, 24H, $24 \mathrm{Ar}-\mathrm{H}) .{ }^{13} \mathrm{C}$ NMR and HSQC $\left(100 \mathrm{MHz}, \mathrm{CDCl}_{3}\right) \delta_{\mathrm{C}} 31.6(2 \mathrm{C}-13), 41.3(2 \mathrm{C}-6)$, 45.4 (2C-12), $52.4\left(2 \mathrm{CH}_{3}\right), 52.4(2 \mathrm{C}-3), 54.1\left(2 \mathrm{OCH}_{3}\right), 54.2(2 \mathrm{C}-15), 62.8(2 \mathrm{C}-10), 67.3(2 \mathrm{C}-9), 69.7$ (2C-2), 101.7 (2C-7), 102.2 (2C-14), 127.0-128.9 (24 Ar-CH), 138.3 (2 Ar-C), 138.5 (2 Ar-C), 139.6 (2 Ar-C), 168.7 (2CO). MS (ESI $\left.{ }^{+}\right): m / z(\%) 981(23)[\mathrm{M}+\mathrm{Na}]^{+}, 959(100)[\mathrm{M}+\mathrm{H}]^{+}, 613(14), 499$ (23), 491 (10). HRMS (ESI ${ }^{+}$): calcd. for $\mathrm{C}_{56} \mathrm{H}_{71} \mathrm{~N}_{4} \mathrm{O}_{10}[\mathrm{M}+\mathrm{H}]^{+}$959.5170, found 959.5214 .

Data for 16c: $R_{\mathrm{f}}=0.66\left(\mathrm{Et}_{2} \mathrm{O} / \mathrm{MeOH}, 98: 2\right)$. $[\alpha]_{\mathrm{D}}{ }^{20}-77.2\left(c 0.23, \mathrm{CHCl}_{3}\right)$. IR $\left(\mathrm{CHCl}_{3}\right): v_{\max } 3435$ and $3360(\mathrm{NH}), 1666(\mathrm{CO}) .{ }^{1} \mathrm{H}$ NMR and $\operatorname{COSY}{ }^{1} \mathrm{H}-{ }^{1} \mathrm{H}\left(400 \mathrm{MHz}, \mathrm{CDCl}_{3}\right): \delta_{\mathrm{H}} 1.85(\mathrm{~m}, 2 \mathrm{H}, \mathrm{H}-13 \mathrm{~A}, \mathrm{H}-13 \mathrm{~B})$, $2.60\left(\mathrm{dd}, 1 \mathrm{H}, \mathrm{H}-6 \mathrm{~B},{ }^{2} J=14.6 \mathrm{~Hz},{ }^{3} J=2.6 \mathrm{~Hz}\right), 2.66\left(\mathrm{dd}, 1 \mathrm{H}, \mathrm{H}-6 \mathrm{~A},{ }^{2} J=14.6 \mathrm{~Hz},{ }^{3} J=6.8 \mathrm{~Hz}\right), 2.74(\mathrm{ddd}$, $\left.1 \mathrm{H}, \mathrm{H}-12 \mathrm{~B},{ }^{2} J=13.3 \mathrm{~Hz},{ }^{3} J=6.8,5.7 \mathrm{~Hz}\right), 3.15$ (ddd, $\left.1 \mathrm{H}, \mathrm{H}-12 \mathrm{~A},{ }^{2} J=13.3 \mathrm{~Hz},{ }^{3} J=7.5,5.6 \mathrm{~Hz}\right), 3.18(\mathrm{~s}$, $\left.3 \mathrm{H}, \mathrm{OCH}_{3}\right), 3.24\left(\mathrm{~s}, 3 \mathrm{H}, \mathrm{OCH}_{3}\right), 3.45\left(\mathrm{~d}, 1 \mathrm{H}, \mathrm{H}-15 \mathrm{~B},{ }^{2} J=14.7 \mathrm{~Hz}\right), 3.74\left(\mathrm{~d}, 1 \mathrm{H}, \mathrm{H}-15 \mathrm{~A},{ }^{2} J=14.7 \mathrm{~Hz}\right)$, $3.79\left(\mathrm{dd}, 1 \mathrm{H}, \mathrm{H}-9 \mathrm{~B},{ }^{2} J=10.5 \mathrm{~Hz},{ }^{3} J=2.9 \mathrm{~Hz}\right), 3.83\left(\mathrm{dd}, \mathrm{H}, \mathrm{H}-2 \mathrm{~B},{ }^{2} J=10.7 \mathrm{~Hz},{ }^{3} J=3.6 \mathrm{~Hz}\right), 3.91(\mathrm{dd}$, $\left.1 \mathrm{H}, \mathrm{H}-10,{ }^{3} J=7.7,2.9 \mathrm{~Hz}\right), 3.92\left(\mathrm{dd}, 1 \mathrm{H}, \mathrm{H}-2 \mathrm{~A},{ }^{2} J=10.7 \mathrm{~Hz},{ }^{3} J=4.9 \mathrm{~Hz}\right), 4.25(\mathrm{dd}, 1 \mathrm{H}, \mathrm{H}-9 \mathrm{~A}$, $\left.{ }^{2} J=10.5 \mathrm{~Hz},{ }^{3} J=7.7 \mathrm{~Hz}\right), 4.51\left(\mathrm{~s}, 2 \mathrm{H}, \mathrm{CH}_{2} \mathrm{Br}\right), 4.77$ (dd, $\left.1 \mathrm{H}, \mathrm{H}-14,{ }^{3} J=6.6,4.0 \mathrm{~Hz}\right), 4.78(\mathrm{dd}, 1 \mathrm{H}, \mathrm{H}-7$, ${ }^{3} J=6.8,2.6 \mathrm{~Hz}$ ), 5.24 (ddd, $\left.1 \mathrm{H}, \mathrm{H}-3,{ }^{3} J=7.7,4.9,3.6 \mathrm{~Hz}\right), 7.15$ (d, $1 \mathrm{H}, \mathrm{NH}$ lactam, $\left.{ }^{3} J=7.7 \mathrm{~Hz}\right), 7.28-$ $7.40(\mathrm{~m}, 14 \mathrm{H}, 14 \mathrm{Ar}-\mathrm{H}) .{ }^{13} \mathrm{C}$ NMR and HSQC (100 MHz, CDCl $), \mathrm{HMBC}\left(125 \mathrm{MHz}, \mathrm{CDCl}_{3}\right): \delta 31.7(\mathrm{C}-$ 
13), $33.7\left(\mathrm{CH}_{2} \mathrm{Br}\right), 41.2(\mathrm{C}-6), 45.3(\mathrm{C}-12), 52.5(\mathrm{C}-3), 52.6\left(\mathrm{OCH}_{3}\right), 54.2(\mathrm{C}-15), 53.3\left(\mathrm{OCH}_{3}\right), 63.1(\mathrm{C}-$ 10), 67.3 (C-9), 69.8 (C-2), 101.8 (C-7), 102.2 (C-14), 127.0-129.2 (14 Ar-CH), 136.4 (Ar-C), 138.0 (ArC), $139.6(\mathrm{Ar}-\mathrm{C}), 140.6(\mathrm{Ar}-\mathrm{C}), 168.6(\mathrm{CO}) . \mathrm{MS}\left(\mathrm{ESI}^{+}\right): \mathrm{m} / z(\%)=635(29)\left[\mathrm{M}+\mathrm{Na}\left({ }^{81} \mathrm{Br}\right)\right]^{+}, 633(28)$ $\left[\mathrm{M}+\mathrm{Na}\left({ }^{79} \mathrm{Br}\right)\right]^{+}, 613(100)\left[\mathrm{M}+\mathrm{H}\left({ }^{81} \mathrm{Br}\right)\right]^{+}, 611(98)\left[\mathrm{M}+\mathrm{H}\left({ }^{79} \mathrm{Br}\right)\right]^{+}$. HRMS $\left(\mathrm{ESI}^{+}\right)$: calcd. for $\mathrm{C}_{32} \mathrm{H}_{40}$ ${ }^{79} \mathrm{BrN}_{2} \mathrm{O}_{5}[\mathrm{M}+\mathrm{H}]^{+}$611.2121, found 611.2131 .

6.1.3.3. Bis-(3S,7S,10S,14R)-dimethyl diketal aminolactam $(14 b)$ and $(3 S, 7 S, 10 S, 14 R)$-bromoxylyl dimethyl diketal aminolactam $(17 b)$

These compounds were prepared from dimethyl diketal aminolactam $\mathbf{5 b}(15.2 \mathrm{mg}, 50.0 \mu \mathrm{mol}), \alpha, \alpha^{\prime}-$ dibromo- $p$-xylene (7.9 mg, $30.0 \mu \mathrm{mol}, 0.6$ equiv.), $\mathrm{K}_{2} \mathrm{CO}_{3}\left(8.3 \mathrm{mg}, 60.0 \mu \mathrm{mol}, 1.2\right.$ equiv.), and $\mathrm{CH}_{3} \mathrm{CN}$ $\left(1.0 \mathrm{~mL}, c_{\text {substrate }}=50 \times 10^{-3} \mathrm{M}\right)$ according to the general procedure (reaction time $\left.9 \mathrm{~h}\right)$. Chromatography $\left(\mathrm{Et}_{2} \mathrm{O} / \mathrm{MeOH}\right.$, gradient 100:0-50:50) afforded bis-dimethyl diketal aminolactam 14b (11.5 mg, $16.2 \mu \mathrm{mol}, 72 \%)$ and bromoxylyl dimethyl diketal aminolactam 17b (1.3 mg, $2.7 \mu \mathrm{mol}, 6 \%)$ with 90\% conversion rate.

Data for 14b: $R_{\mathrm{f}}=0.35\left(\mathrm{Et}_{2} \mathrm{O} / \mathrm{MeOH}, 50: 50\right), 0.45\left(\mathrm{MeOH} / \mathrm{NH}_{4} \mathrm{OH}, 99: 1\right)$. $[\alpha]_{\mathrm{D}}{ }^{25}+55.2\left(c 0.69, \mathrm{CHCl}_{3}\right)$. IR $\left(\mathrm{CHCl}_{3}\right): v 3365(\mathrm{NH}), 1653(\mathrm{CO}) .{ }^{1} \mathrm{H} \mathrm{NMR}, \mathrm{COSY}{ }^{1} \mathrm{H}-{ }^{1} \mathrm{H}\left(400 \mathrm{MHz}, \mathrm{CDCl}_{3}\right): \delta_{\mathrm{H}} 1.02\left(\mathrm{~d}, 6 \mathrm{H}, 2 \mathrm{CH}_{3}\right.$, $\left.{ }^{3} J=6.9 \mathrm{~Hz}\right), 1.24\left(\mathrm{~d}, 6 \mathrm{H}, 2 \mathrm{CH}_{3},{ }^{3} J=6.7 \mathrm{~Hz}\right), 1.86(\mathrm{~m}, 4 \mathrm{H}, 4 \mathrm{H}-13), 2.49$ (dd, 2H, 2H-6B, ${ }^{2} J=16.8 \mathrm{~Hz}$, $\left.{ }^{3} J=1.9 \mathrm{~Hz}\right), 2.53\left(\mathrm{dt}, 2 \mathrm{H}, 2 \mathrm{H}-12 \mathrm{~B},{ }^{2} J=12.5 \mathrm{~Hz},{ }^{3} J=3.9 \mathrm{~Hz}\right), 2.72\left(\mathrm{dd}, 2 \mathrm{H}, 2 \mathrm{H}-6 \mathrm{~A},{ }^{2} J=16.8 \mathrm{~Hz}\right.$, ${ }^{3} J=9.0 \mathrm{~Hz}$ ), 2.90 (qdd, $\left.2 \mathrm{H}, 2 \mathrm{H}-10,{ }^{3} J=6.9,6.3,2.2 \mathrm{~Hz}\right), 3.16\left(\mathrm{ddd}, 2 \mathrm{H}, 2 \mathrm{H}-12 \mathrm{~A},{ }^{2} J=12.5 \mathrm{~Hz},{ }^{3} J=11.5\right.$, $5.1 \mathrm{~Hz}), 3.27\left(\mathrm{~s}, 6 \mathrm{H}, 2 \mathrm{OCH}_{3}\right), 3.32\left(\mathrm{~s}, 6 \mathrm{H}, 2 \mathrm{OCH}_{3}\right), 3.35\left(\mathrm{~d}, 2 \mathrm{H}, 2 \mathrm{H}-15 \mathrm{~B},{ }^{2} \mathrm{~J}=14.3 \mathrm{~Hz}\right), 3.45(\mathrm{dd}, 2 \mathrm{H}$, $2 \mathrm{H}-2 \mathrm{~B},{ }^{2} J=9.7 \mathrm{~Hz},{ }^{3} J=3.1 \mathrm{~Hz}$ ), 3.54 (ABX system, AB part, 4H, 2H-9A, 2H-9B, ${ }^{2} J=10.0 \mathrm{~Hz},{ }^{3} J=6.3$, $2.2 \mathrm{~Hz}, \Delta v=13.4 \mathrm{~Hz}), 3.76\left(\mathrm{dd}, 2 \mathrm{H}, 2 \mathrm{H}-2 \mathrm{~A},{ }^{2} J=9.7 \mathrm{~Hz},{ }^{3} J=2.0 \mathrm{~Hz}\right), 3.81(\mathrm{~d}, 2 \mathrm{H}, 2 \mathrm{H}-15 \mathrm{~A}$, $\left.{ }^{2} J=14.3 \mathrm{~Hz}\right), 4.27(\mathrm{~m}, 2 \mathrm{H}, 2 \mathrm{H}-3), 4.73\left(\mathrm{dd}, 2 \mathrm{H}, 2 \mathrm{H}-7,{ }^{3} J=9.0,1.9 \mathrm{~Hz}\right), 4.81\left(\mathrm{dd}, 2 \mathrm{H}, 2 \mathrm{H}-14,{ }^{3} J=8.7\right.$, $2.6 \mathrm{~Hz}), 7.26\left(\mathrm{~d}, 4 \mathrm{H}, 4 \mathrm{ArH},{ }^{3} \mathrm{~J}=7.1 \mathrm{~Hz}\right), 7.48\left(\mathrm{~d}, 1 \mathrm{H}, \mathrm{NH}\right.$ lactam, $\left.{ }^{3} \mathrm{~J}=8.6 \mathrm{~Hz}\right) .{ }^{13} \mathrm{C} \mathrm{NMR}$ and HSQC $\left(100 \mathrm{MHz}, \mathrm{CDCl}_{3}\right): \delta_{\mathrm{C}} 8.0\left(2 \mathrm{CH}_{3}\right), 18.1\left(2 \mathrm{CH}_{3}\right), 30.8(2 \mathrm{C}-13), 39.4(2 \mathrm{C}-6), 44.4(2 \mathrm{C}-12), 44.6(2 \mathrm{C}-3)$, $51.9\left(2 \mathrm{OCH}_{3}\right), 53.8(2 \mathrm{C}-10), 53.9(2 \mathrm{C}-15), 54.4\left(2 \mathrm{OCH}_{3}\right), 67.8$ (2C-9), $71.4(2 \mathrm{C}-2), 99.7(2 \mathrm{C}-7), 102.3$ (2C-14), 128.4 (4 Ar-CH), 138.8 (2 Ar-C), 167.9 (2CO). MS (APCI $\left.{ }^{+}\right) m / z(\%) 711(100)\left[\mathrm{MH}^{+}\right], 679$ (30) $\left[\mathrm{M}+\mathrm{H}-\mathrm{CH}_{3} \mathrm{OH}\right]^{+}, 647$ (7). HRMS $\left(\mathrm{ESI}^{+}\right)$: calcd. for $\mathrm{C}_{36} \mathrm{H}_{63} \mathrm{~N}_{4} \mathrm{O}_{10}[\mathrm{M}+\mathrm{H}]^{+}$711.4544, found 711.4576 .

Data for 17b: $R_{\mathrm{f}}=0.55\left(\mathrm{Et}_{2} \mathrm{O} / \mathrm{MeOH}, 50: 50\right), 0.75\left(\mathrm{MeOH} / \mathrm{NH}_{4} \mathrm{OH}, 99: 1\right)$. $[\alpha]_{\mathrm{D}}{ }^{25}+19.0\left(c 0.65, \mathrm{CHCl}_{3}\right)$. IR $\left(\mathrm{CHCl}_{3}\right): v_{\max } 3365$ and $3290(\mathrm{NH}), 1662(\mathrm{CO}) .{ }^{1} \mathrm{H}$ NMR and $\mathrm{COSY}{ }^{1} \mathrm{H}-{ }^{1} \mathrm{H}\left(400 \mathrm{MHz}, \mathrm{CDCl}_{3}\right): \delta_{\mathrm{H}}$ $1.02\left(\mathrm{~d}, 3 \mathrm{H}, \mathrm{CH}_{3},{ }^{3} \mathrm{~J}=6.8 \mathrm{~Hz}\right), 1.25$ (d, 3H, $\left.\mathrm{CH}_{3},{ }^{3} \mathrm{~J}=6.8 \mathrm{~Hz}\right), 1.84$ (m, 2H, H-13A, H-13B), 2.50 (dd, $\left.1 \mathrm{H}, \mathrm{H}-6 \mathrm{~B},{ }^{2} J=16.6 \mathrm{~Hz},{ }^{3} J=1.9 \mathrm{~Hz}\right), 2.54\left(\mathrm{dt}, 1 \mathrm{H}, \mathrm{H}-12 \mathrm{~B},{ }^{2} J=12.5 \mathrm{~Hz},{ }^{3} J=4.1 \mathrm{~Hz}\right), 2.73(\mathrm{dd}, 1 \mathrm{H}, \mathrm{H}-$ $\left.6 \mathrm{~A},{ }^{2} J=16.6 \mathrm{~Hz},{ }^{3} J=9.0 \mathrm{~Hz}\right), 2.92\left(\mathrm{dqd}, 1 \mathrm{H}, \mathrm{H}-10,{ }^{3} J=6.8,6.0,2.0 \mathrm{~Hz}\right), 3.15(\mathrm{ddd}, 1 \mathrm{H}, \mathrm{H}-12 \mathrm{~A}$, $\left.{ }^{2} J=12.5 \mathrm{~Hz},{ }^{3} J=11.0,6.1 \mathrm{~Hz}\right), 3.25\left(\mathrm{~s}, 3 \mathrm{H}, \mathrm{OCH}_{3}\right), 3.31\left(\mathrm{~s}, 3 \mathrm{H}, \mathrm{OCH}_{3}\right), 3.41(\mathrm{~d}, 1 \mathrm{H}, \mathrm{H}-15 \mathrm{~B}$, ${ }^{2} J=14.7 \mathrm{~Hz}$ ), $3.44\left(\mathrm{dd}, 1 \mathrm{H}, \mathrm{H}-2 \mathrm{~B},{ }^{2} J=10.0 \mathrm{~Hz},{ }^{3} J=2.8 \mathrm{~Hz}\right), 3.55$ (ABX, AB part, 2H, H-9A, H-9B, $\left.{ }^{2} J=10.0,{ }^{3} J=6.0,2.0 \mathrm{~Hz}, \Delta v=11.1 \mathrm{~Hz}\right), 3.75\left(\mathrm{dd}, 1 \mathrm{H}, \mathrm{H}-2 \mathrm{~A},{ }^{2} J=10.0 \mathrm{~Hz},{ }^{3} J=1.8 \mathrm{~Hz}\right), 3.81(\mathrm{~d}, 1 \mathrm{H}, \mathrm{H}-$ $\left.15 \mathrm{~A},{ }^{2} J=14.7 \mathrm{~Hz}\right), 4.26(\mathrm{~m}, 1 \mathrm{H}, \mathrm{H}-3), 4.50\left(\mathrm{~s}, 2 \mathrm{H}, \mathrm{CH}_{2} \mathrm{Br}\right), 4.71$ (dd, $\left.1 \mathrm{H}, \mathrm{H}-7,{ }^{3} J=9.0,1.9 \mathrm{~Hz}\right), 4.75$ (dd, $\left.1 \mathrm{H}, \mathrm{H}-14,{ }^{3} J=7.8,3.2 \mathrm{~Hz}\right), 7.26\left(\mathrm{~d}, 4 \mathrm{H}, 4 \mathrm{Ar}-\mathrm{H},{ }^{3} J=7.1 \mathrm{~Hz}\right), 7.46$ (d, 1H, NH lactam, $\left.{ }^{3} J=8.1 \mathrm{~Hz}\right)$.

${ }^{13} \mathrm{C}$ NMR and HSQC $\left(100 \mathrm{MHz}, \mathrm{CDCl}_{3}\right) \delta_{\mathrm{C}} 8.1\left(\mathrm{CH}_{3}\right), 18.1\left(\mathrm{CH}_{3}\right), 30.9(\mathrm{C}-13), 33.6\left(\mathrm{CH}_{2} \mathrm{Br}\right), 39.5(\mathrm{C}-6)$, $44.3(\mathrm{C}-12), 44.5(\mathrm{C}-3), 52.0\left(\mathrm{OCH}_{3}\right), 53.0(\mathrm{C}-10), 54.0(\mathrm{C}-15), 54.3\left(\mathrm{OCH}_{3}\right), 67.7(\mathrm{C}-2), 71.4(\mathrm{C}-9), 99.7$ (C-7), 102.3 (C-14), 128.5-129.2 (4 Ar-CH), 137.1 (Ar-C), 145.0 (Ar-C), 168.0 (CO). MS (APCI $\left.{ }^{+}\right): m / z$ (\%) 489 (92) $\left[\mathrm{M}+\mathrm{H}\left({ }^{81} \mathrm{Br}\right)\right]^{+}, 487$ (79) $\left[\mathrm{M}+\mathrm{H}\left({ }^{79} \mathrm{Br}\right)\right]^{+}, 457$ (57) $\left[\mathrm{M}+\mathrm{H}-\mathrm{CH}_{3} \mathrm{OH}\left({ }^{81} \mathrm{Br}\right)\right]^{+}, 455$ (36) $\left[\mathrm{M}+\mathrm{H}-\mathrm{CH}_{3} \mathrm{OH}\left({ }^{79} \mathrm{Br}\right)\right]^{+}, 431(100), 429(91), 385$ (15), 127 (49). HRMS (ESI $\left.{ }^{+}\right)$: calcd for $\mathrm{C}_{22} \mathrm{H}_{36}$ ${ }^{79} \mathrm{BrN}_{2} \mathrm{O}_{5}[\mathrm{M}+\mathrm{H}]^{+}$487.1807, found 487.1826.

\subsubsection{Bis- $\left(7 S^{*}, 14 R^{*}\right)$-diketal aminolactam $(15 \mathrm{~b})$}

This compound was prepared from diketal aminolactam $\mathbf{6 b}(13.8 \mathrm{mg}, 50.0 \mu \mathrm{mol}), \alpha, \alpha^{\prime}$-dibromo- $p$-xylene (6.6 mg, $25.0 \mu \mathrm{mol}, 0.5$ equiv.), $\mathrm{K}_{2} \mathrm{CO}_{3}\left(6.9 \mathrm{mg}, 50.0 \mu \mathrm{mol}, 1.0\right.$ equiv.), and $\mathrm{CH}_{3} \mathrm{CN}(1.0 \mathrm{~mL}$, $c_{\text {substrate }}=50 \times 10^{-3} \mathrm{M}$ ) according to the general procedure (reaction time $9 \mathrm{~h}$ ). Chromatography $\left(\mathrm{Et}_{2} \mathrm{O} / \mathrm{MeOH}\right.$, gradient 100:0-0:100) afforded bis-diketal aminolactam 15b (6.6 mg, $\left.10.1 \mu \mathrm{mol}, 48 \%\right)$ with $84 \%$ conversion rate. $R_{\mathrm{f}}=0.38\left(\mathrm{MeOH} / \mathrm{NH}_{4} \mathrm{OH}, 99: 1\right)$. IR $\left(\mathrm{CHCl}_{3}\right): v_{\max } 3450$ and $3369(\mathrm{NH}), 1656$ (CO). ${ }^{1} \mathrm{H}$ NMR and COSY ${ }^{1} \mathrm{H}-{ }^{1} \mathrm{H}\left(400 \mathrm{MHz}, \mathrm{CDCl}_{3}\right): \delta_{\mathrm{H}} 1.91(\mathrm{~m}, 4 \mathrm{H}, 4 \mathrm{H}-13), 2.48(\mathrm{~m}, 2 \mathrm{H}, 2 \mathrm{H}-10 \mathrm{~B})$, $2.52\left(\mathrm{dd}, 2 \mathrm{H}, 2 \mathrm{H}-6 \mathrm{~B},{ }^{2} J=15.1 \mathrm{~Hz},{ }^{3} \mathrm{~J}=1.0 \mathrm{~Hz}\right), 2.58(\mathrm{~m}, 2 \mathrm{H}, 2 \mathrm{H}-12 \mathrm{~B}), 2.66$ (dd, 2H, 2H-6A, 
$\left.{ }^{2} J=15.1 \mathrm{~Hz},{ }^{3} J=7.8 \mathrm{~Hz}\right), 2.68(\mathrm{~m}, 2 \mathrm{H}, 2 \mathrm{H}-10 \mathrm{~A}), 2.88(\mathrm{~m}, 2 \mathrm{H}, 2 \mathrm{H}-12 \mathrm{~A}), 3.30\left(\mathrm{~s}, 6 \mathrm{H}, 2 \mathrm{OCH}_{3}\right), 3.31$ (s, $\left.6 \mathrm{H}, 2 \mathrm{OCH}_{3}\right), 3.42\left(\mathrm{~d}, 2 \mathrm{H}, 2 \mathrm{H}-15 \mathrm{~B},{ }^{2} \mathrm{~J}=15.0 \mathrm{~Hz}\right), 3.50(\mathrm{~m}, 4 \mathrm{H}, 4 \mathrm{H}-3), 3.51(\mathrm{~m}, 2 \mathrm{H}, 2 \mathrm{H}-2 \mathrm{~B}), 3.57$ (ddd, $\left.2 \mathrm{H}, 2 \mathrm{H}-9 \mathrm{~B},{ }^{2} \mathrm{~J}=9.0 \mathrm{~Hz},{ }^{3} \mathrm{~J}=8.4,1.0 \mathrm{~Hz}\right), 3.70\left(\mathrm{~d}, 2 \mathrm{H}, 2 \mathrm{H}-15 \mathrm{~A},{ }^{2} J=15.0 \mathrm{~Hz}\right), 3.73(\mathrm{~m}, 2 \mathrm{H}, 2 \mathrm{H}-9 \mathrm{~A}), 3.78$ $(\mathrm{m}, 2 \mathrm{H}, 2 \mathrm{H}-2 \mathrm{~A}), 4.70\left(\mathrm{dd}, 2 \mathrm{H}, 2 \mathrm{H}-7,{ }^{3} J=7.8,1.0 \mathrm{~Hz}\right), 4.73\left(\mathrm{dd}, 2 \mathrm{H}, 2 \mathrm{H}-14,{ }^{3} \mathrm{~J}=10.4,5.2 \mathrm{~Hz}\right), 7.25$ (br. s, 2H, $2 \mathrm{NH}$ lactam), $7.26\left(\mathrm{~d}, 4 \mathrm{H}, 4 \mathrm{Ar}-\mathrm{H},{ }^{3} \mathrm{~J}=7.8 \mathrm{~Hz}\right) .{ }^{13} \mathrm{C} \mathrm{NMR}$ and HSQC $\left(100 \mathrm{MHz}, \mathrm{CDCl}_{3},\right): \delta_{\mathrm{C}} 30.2$ (2C-13), 38.8 (2C-3), 40.5 (2C-6), 49.9 (2C-12), $52.5\left(2 \mathrm{OCH}_{3}\right), 53.4\left(2 \mathrm{OCH}_{3}\right), 54.0(2 \mathrm{C}-10), 57.9(2 \mathrm{C}-$ 15), 64.7 (2C-2), 65.2 (2C-9), 100.4 (2C-7), 101.4 (2C-14), 129.0 (4 Ar-CH), 140.3 (2 Ar-C), 169.0 (2CO). MS (ESI $\left.{ }^{+}\right): m / z(\%) 693(5)[\mathrm{M}+\mathrm{K}]^{+}, 677(76)[\mathrm{M}+\mathrm{Na}]^{+}, 655(100)[\mathrm{M}+\mathrm{H}]^{+}, 623(4)[\mathrm{M}+\mathrm{H}-$ $\left.\mathrm{CH}_{3} \mathrm{OH}\right]^{+}$. HRMS (ESI $\left.{ }^{+}\right)$: calcd. for $\mathrm{C}_{32} \mathrm{H}_{55} \mathrm{~N}_{4} \mathrm{O}_{10}[\mathrm{M}+\mathrm{H}]^{+} 655.3918$, found 655.3931 .

\subsubsection{Bis-(7R*,14R*)-diketal aminolactam (15c)}

This compound was prepared from diketal aminolactam $6 \mathbf{c}(16.6 \mathrm{mg}, 60.0 \mu \mathrm{mol}), \alpha, \alpha^{\prime}$-dibromo- $p$-xylene (9.5 mg, $36.0 \mu \mathrm{mol}$, 0.6 equiv.), $\mathrm{K}_{2} \mathrm{CO}_{3}\left(10.0 \mathrm{mg}, 72.0 \mu \mathrm{mol}, 1.2\right.$ equiv.), and $\mathrm{CH}_{3} \mathrm{CN}(1.2 \mathrm{~mL}$, $c_{\text {substrate }}=50 \times 10^{-3} \mathrm{M}$ ) according to the general procedure (reaction time $9 \mathrm{~h}$ ). Chromatography $\left(\mathrm{Et}_{2} \mathrm{O} / \mathrm{MeOH}\right.$, gradient 100:0-0:100) afforded bis-diketal aminolactam 15c $(9.4 \mathrm{mg}, 14.3 \mu \mathrm{mol}, 55 \%)$ with $87 \%$ conversion rate. $R_{\mathrm{f}}=0.35(\mathrm{MeOH})$. IR $\left(\mathrm{CHCl}_{3}\right): v_{\max } 3450$ and $3350(\mathrm{NH}), 1660(\mathrm{CO}) .{ }^{1} \mathrm{H}$ NMR and COSY ${ }^{1} \mathrm{H}^{1}{ }^{1} \mathrm{H}\left(400 \mathrm{MHz}, \mathrm{CDCl}_{3}\right): \delta_{\mathrm{H}} 1.88(\mathrm{~m}, 4 \mathrm{H}, 4 \mathrm{H}-13), 2.53(\mathrm{~m}, 6 \mathrm{H}, 2 \mathrm{H}-6 \mathrm{~B}, 2 \mathrm{H}-10 \mathrm{~B}, 2 \mathrm{H}-$ 12B), 2.64 (m, 4H, 2H-6A, 2H-10A), 2.90 (dt, 2H, $\left.2 \mathrm{H}-12 \mathrm{~A},{ }^{2} J=12.0 \mathrm{~Hz},{ }^{3} J=6.0 \mathrm{~Hz}\right), 3.21$ (dddd, $2 \mathrm{H}$, $\left.2 \mathrm{H}-3 \mathrm{~B},{ }^{2} J=11.2 \mathrm{~Hz},{ }^{3} J=7.7,3.0,1.0 \mathrm{~Hz}\right), 3.30\left(\mathrm{~s}, 12 \mathrm{H}, 4 \mathrm{OCH}_{3}\right), 3.40\left(\mathrm{~d}, 2 \mathrm{H}, 2 \mathrm{H}-15 \mathrm{~B},{ }^{2} J=14.0 \mathrm{~Hz}\right)$, 3.50 (ddd, $2 \mathrm{H}, 2 \mathrm{H}-9 \mathrm{~B},{ }^{2} J=8.1 \mathrm{~Hz},{ }^{3} J=7.3 \mathrm{~Hz}, 1.0 \mathrm{~Hz}$ ), 3.57 (ddd, $2 \mathrm{H}, 2 \mathrm{H}-2 \mathrm{~B},{ }^{2} J=9.0 \mathrm{~Hz},{ }^{3} J=8.4$, $1.0 \mathrm{~Hz}), 3.72\left(\mathrm{~d}, 2 \mathrm{H}, 2 \mathrm{H}-15 \mathrm{~A},{ }^{2} J=14.0 \mathrm{~Hz}\right), 3.76(\mathrm{~m}, 4 \mathrm{H}, 2 \mathrm{H}-2 \mathrm{~A}, 2 \mathrm{H}-9 \mathrm{~A}), 3.81$ (m, 2H, 2H-3A), 4.66 (dd, 2H, 2H-7, $\left.{ }^{3} \mathrm{~J}=6.8,1.0 \mathrm{~Hz}\right), 4.72\left(\mathrm{t}, 2 \mathrm{H}, 2 \mathrm{H}-14,{ }^{3} \mathrm{~J}=5.2 \mathrm{~Hz}\right), 7.16$ (br. s, $2 \mathrm{H}, 2 \mathrm{NH}$ lactam), 7.26 (d, $\left.4 \mathrm{H}, 4 \mathrm{ArH},{ }^{3} \mathrm{~J}=8.0 \mathrm{~Hz}\right) .{ }^{13} \mathrm{C} \mathrm{NMR}$ and HSQC $\left(100 \mathrm{MHz}, \mathrm{CDCl}_{3}\right): \delta_{\mathrm{C}} 30.9(2 \mathrm{C}-13), 39.2(2 \mathrm{C}-3), 40.8$ (2C-6), $50.2(2 \mathrm{C}-12), 52.6\left(2 \mathrm{OCH}_{3}\right), 53.6\left(2 \mathrm{OCH}_{3}\right), 54.1(2 \mathrm{C}-10), 58.2(2 \mathrm{C}-15), 66.0(2 \mathrm{C}-9,2 \mathrm{C}-2)$, 100.9 (2C-7), 102.2 (2C-14), 129.1 (4 Ar-CH), 140.2 (2C-16), 169.1 (2CO). MS (ESI $\left.{ }^{+}\right): m / z(\%) 693$ (19) $[\mathrm{M}+\mathrm{K}]^{+}, 677(8)[\mathrm{M}+\mathrm{Na}]^{+}, 655(100)[\mathrm{M}+\mathrm{H}]^{+}, 627$ (4) $\left[\mathrm{M}+\mathrm{H}-\mathrm{CH}_{3} \mathrm{OH}\right]^{+}$. HRMS $\left(\mathrm{ESI}^{+}\right)$: calcd. for $\mathrm{C}_{32} \mathrm{H}_{55} \mathrm{~N}_{4} \mathrm{O}_{10}[\mathrm{M}+\mathrm{H}]^{+}$655.3918, found 655.3887.

\subsubsection{Bis-diketal diamines: general procedure}

To a solution of bis-diketal aminolactam (1 equiv.) in dry THF $\left(c_{\text {substrate }}=60-100 \times 10^{-3} \mathrm{M}\right)$, was added, dropwise while stirring, a solution of $\mathrm{LiAlH}_{4}$ (1 M solution in THF, 10 equiv.) and $\mathrm{NEt}_{3}$ ( 0.02 equiv./LAH). The reaction mixture was heated at reflux for 6-9 h. Excess hydride was destroyed by adding $\mathrm{H}_{2} \mathrm{O}$ (10 equiv./LAH). The precipitates were filtered and washed with THF and $\mathrm{CH}_{2} \mathrm{Cl}_{2}$. The organic layer was dried with $\mathrm{MgSO}_{4}$ and evaporated in vacuo. Purification by flash chromatography (silicagel, $\mathrm{Et}_{2} \mathrm{O} / \mathrm{MeOH}$ and $\mathrm{MeOH} / \mathrm{NH}_{4} \mathrm{OH}$ ) yielded bis-diketal diamines.

\subsubsection{Bis-(3S,7S,10S,14R)-dimethyl diketal diamine (18b)}

This compound was prepared from bis-dimethyl diketal aminolactam 14b $(11.4 \mathrm{mg}, 16 \mu \mathrm{mol}), \mathrm{LiAlH}_{4}$ ( $1 \mathrm{M}$ THF solution, $160 \mu \mathrm{L}, 160 \mu \mathrm{mol}, 10$ equiv.), $\mathrm{NEt}_{3}(1 \mathrm{M}$ THF solution, $3.2 \mu \mathrm{L}, 3.2 \mu \mathrm{mol}$, 0.02 equiv./LAH), THF $\left(160 \mu \mathrm{L}, c_{\text {substrate }}=100 \times 10^{-3} \mathrm{M}\right)$ according to the general procedure (reaction time $9 \mathrm{~h})$. Excess hydride was destroyed by adding $\mathrm{H}_{2} \mathrm{O}(29 \mu \mathrm{L}, 1.6 \mathrm{mmol})$. Chromatography $\left(\mathrm{Et}_{2} \mathrm{O} / \mathrm{MeOH}\right.$, gradient 100:0-0:100 and $\mathrm{MeOH} / \mathrm{NH}_{4} \mathrm{OH}$, gradient 100:0-90:10) afforded bis-dimethyl diketal diamine $\mathbf{1 8 b}(2.9 \mathrm{mg}, 4.2 \mu \mathrm{mol}, 26 \%)$ and dimethyl diketal diamine $\mathbf{8 b}(0.8 \mathrm{mg}, 2.8 \mu \mathrm{mol}, 9 \%)$.

Data for 18b: $R_{\mathrm{f}}=0.20\left(\mathrm{MeOH} / \mathrm{NH}_{4} \mathrm{OH}, 97: 3\right)$. IR $\left(\mathrm{CHCl}_{3}\right): v_{\max } 3340(\mathrm{NH}) .{ }^{1} \mathrm{H}$ NMR and COSY ${ }^{1} \mathrm{H}-{ }^{1} \mathrm{H}$ $\left(400 \mathrm{MHz}, \mathrm{CDCl}_{3}\right): \delta_{\mathrm{H}} 1.01\left(\mathrm{~d}, 6 \mathrm{H}, 2 \mathrm{CH}_{3},{ }^{3} \mathrm{~J}=6.8 \mathrm{~Hz}\right), 1.10\left(\mathrm{~d}, 6 \mathrm{H}, 2 \mathrm{CH}_{3},{ }^{3} J=6.6 \mathrm{~Hz}\right), 1.81(\mathrm{~m}, 4 \mathrm{H}, 4 \mathrm{H}-$ 13), 1.92 (m, 4H, 4H-6), 2.05 (br. s, $2 \mathrm{H}, 2 \mathrm{NH}$ amine), 2.53 (dt, 2H, $\left.2 \mathrm{H}-12 \mathrm{~B},{ }^{2} \mathrm{~J}=12.5 \mathrm{~Hz},{ }^{3} \mathrm{~J}=5.1 \mathrm{~Hz}\right)$, 2.70 (m, 2H, 2H-5B), 2.90 (m, 6H, 2H-3, 2H-5A, 2H-10), 3.07 (ddd, $2 \mathrm{H}, 2 \mathrm{H}-12 \mathrm{~A},{ }^{2} \mathrm{~J}=12.6 \mathrm{~Hz},{ }^{3} \mathrm{~J}=9.5$, $6.2 \mathrm{~Hz}), 3.28\left(\mathrm{~s}, 6 \mathrm{H}, 2 \mathrm{OCH}_{3}\right), 3.32\left(\mathrm{~s}, 6 \mathrm{H}, 2 \mathrm{OCH}_{3}\right), 3.45\left(\mathrm{~d}, 2 \mathrm{H}, 2 \mathrm{H}-15 \mathrm{~B},{ }^{2} J=14.4 \mathrm{~Hz}\right), 3.46(\mathrm{dd}, 2 \mathrm{H}$, $\left.2 \mathrm{H}-2 \mathrm{~B},{ }^{2} J=10.0 \mathrm{~Hz},{ }^{3} \mathrm{~J}=2.4 \mathrm{~Hz}\right), 3.48\left(\mathrm{td}, 2 \mathrm{H}, 2 \mathrm{H}-9 \mathrm{~B},{ }^{2} J=10.0 \mathrm{~Hz},{ }^{3} J=6.0 \mathrm{~Hz}\right), 3.61(\mathrm{td}, 2 \mathrm{H}, 2 \mathrm{H}-9 \mathrm{~A}$, $\left.{ }^{2} J=10.0 \mathrm{~Hz},{ }^{3} J=2.5 \mathrm{~Hz}\right), 3.66\left(\mathrm{dd}, 2 \mathrm{H}, 2 \mathrm{H}-2 \mathrm{~A},{ }^{2} J=10.0 \mathrm{~Hz},{ }^{3} J=2.3 \mathrm{~Hz}\right), 3.75(\mathrm{~d}, 2 \mathrm{H}, 2 \mathrm{H}-15 \mathrm{~A}$, $\left.{ }^{2} J=14.4 \mathrm{~Hz}\right), 4.58\left(\mathrm{dd}, 2 \mathrm{H}, 2 \mathrm{H}-7,{ }^{3} J=6.4,3.8 \mathrm{~Hz}\right), 4.77\left(\mathrm{dd}, 2 \mathrm{H}, 2 \mathrm{H}-14,{ }^{3} \mathrm{~J}=7.1,1.8 \mathrm{~Hz}\right), 7.28(\mathrm{~d}, 4 \mathrm{H}, 4$ $\left.\mathrm{ArH},{ }^{3} J=6.8 \mathrm{~Hz}\right) .{ }^{13} \mathrm{C} \mathrm{NMR}$ and HSQC $\left(100 \mathrm{MHz}, \mathrm{CDCl}_{3}\right)$, HMBC $\left(125 \mathrm{MHz}, \mathrm{CDCl}_{3}\right): \delta_{\mathrm{C}} 9.7\left(2 \mathrm{CH}_{3}\right)$, 
$16.0\left(2 \mathrm{CH}_{3}\right), 32.0(2 \mathrm{C}-6,2 \mathrm{C}-13), 42.2(2 \mathrm{C}-5), 44.4(2 \mathrm{C}-12), 52.8(2 \mathrm{C}-3), 52.9\left(2 \mathrm{OCH}_{3}\right), 54.3\left(2 \mathrm{OCH}_{3}\right)$, 54.5 (2C-10), 54.6 (2C-15), 68.3 (2C-2), 70.0 (2C-9), 101.8 (2C-14), 103.4 (2C-7), 128.3 (4 Ar-CH), 139.2 (2 Ar-C). MS (APCI) $m / z(\%): 705$ (9) $[\mathrm{M}+\mathrm{Na}]^{+}, 683(100)[\mathrm{M}+\mathrm{H}]^{+}, 655$ (7), 613 (8). HRMS $\left(\mathrm{ESI}^{+}\right.$): calcd. for $\mathrm{C}_{36} \mathrm{H}_{67} \mathrm{~N}_{4} \mathrm{O}_{8}[\mathrm{M}+\mathrm{H}]^{+}$683.4959, found 683.4955 .

\subsubsection{Bis-( $\left.7 R^{*}, 14 R^{*}\right)$-diketal diamine (19c)}

This compound was prepared from bis-diketal aminolactam 15c $(10.5 \mathrm{mg}, 16 \mu \mathrm{mol}), \mathrm{LiAlH}_{4}(1 \mathrm{M}$ THF solution, $160 \mu \mathrm{L}, 160 \mu \mathrm{mol}, 10$ equiv.), $\mathrm{NEt}_{3}$ ( $1 \mathrm{M}$ THF solution, $3.2 \mu \mathrm{L}, 3.2 \mu \mathrm{mol}, 0.02$ equiv./LAH), THF $\left(267 \mu \mathrm{L}, c_{\text {substrate }}=60 \times 10^{-3} \mathrm{M}\right)$ according to the general procedure (reaction time $\left.6 \mathrm{~h}\right)$. Excess hydride was destroyed by adding $\mathrm{H}_{2} \mathrm{O}(29 \mu \mathrm{L}, 1.6 \mathrm{mmol})$. Chromatography $\left(\mathrm{Et}_{2} \mathrm{O} / \mathrm{MeOH}\right.$, gradient 100:0-0:100 and $\mathrm{MeOH} / \mathrm{NH}_{4} \mathrm{OH}$, gradient 100:0-70:30) afforded bis-diketal diamine 19c (2.2 mg, $3.5 \mu \mathrm{mol}, 22 \%)$ and diketal diamine $9 \mathrm{c}(0.8 \mathrm{mg}, 3.0 \mu \mathrm{mol}, 9 \%)$.

Data for 19c: $R_{\mathrm{f}}=0.25\left(\mathrm{MeOH} / \mathrm{NH}_{4} \mathrm{OH}, 94: 4,2\right.$ elutions). IR $\left(\mathrm{CHCl}_{3}\right): v_{\max } 3300(\mathrm{NH})$. NMR ${ }^{1} \mathrm{H}$ $\left(400 \mathrm{MHz}, \mathrm{CDCl}_{3}\right): \delta_{\mathrm{H}} 1.70-2.10(\mathrm{~m}, 14 \mathrm{H}, 2 \mathrm{H}-6 \mathrm{~B}, 2 \mathrm{H}-13 \mathrm{~B}, 2 \mathrm{H}-6 \mathrm{~A}, 2 \mathrm{H}-13 \mathrm{~A}, 2 \mathrm{H}-5 \mathrm{~B}, 2 \mathrm{H}-12 \mathrm{~B}, 2 \mathrm{NH})$, $2.54(\mathrm{~m}, 4 \mathrm{H}, 2 \mathrm{H}-3 \mathrm{~B}, 2 \mathrm{H}-10 \mathrm{~B}), 2.70(\mathrm{~m}, 4 \mathrm{H}, 2 \mathrm{H}-3 \mathrm{~A}, 2 \mathrm{H}-10 \mathrm{~A}), 3.21\left(\mathrm{~d}, 2 \mathrm{H}, 2 \mathrm{H}-15 \mathrm{~B},{ }^{2} \mathrm{~J}=14.5 \mathrm{~Hz}\right), 3.23$ $(\mathrm{m}, 4 \mathrm{H}, 2 \mathrm{H}-5 \mathrm{~A}, 2 \mathrm{H}-12 \mathrm{~A}), 3.30\left(\mathrm{~s}, 6 \mathrm{H}, 2 \mathrm{OCH}_{3}\right), 3.38\left(\mathrm{~s}, 6 \mathrm{H}, 2 \mathrm{OCH}_{3}\right), 3.60-3.71(\mathrm{~m}, 4 \mathrm{H}, 2 \mathrm{H}-2 \mathrm{~B}, 2 \mathrm{H}-$ 9B), 3.86-3.93 (m, 4H, 2H-2A, 2H-9A), 3.98 (d, 2H, 2H-15A, $\left.{ }^{2} J=14.5 \mathrm{~Hz}\right), 4.51$ (ddd, 2H, 2H-7, $\left.{ }^{3} J=8.0,4.7 \mathrm{~Hz},{ }^{4} J=2.4 \mathrm{~Hz}\right), 4.58\left(\mathrm{ddd}, 2 \mathrm{H}, 2 \mathrm{H}-14,{ }^{3} J=7.4,2.3 \mathrm{~Hz},{ }^{4} J=1.7 \mathrm{~Hz}\right), 7.30(\mathrm{~d}, 4 \mathrm{H}, 4 \mathrm{Ar}-\mathrm{H}$, $\left.{ }^{3} J=6.6 \mathrm{~Hz}\right) .{ }^{13} \mathrm{C}$ NMR $\left(100 \mathrm{MHz}, \mathrm{CDCl}_{3}\right): \delta_{\mathrm{C}} 30.2(2 \mathrm{C}-13), 31.7(2 \mathrm{C}-6), 43.6(2 \mathrm{C}-5), 47.4(2 \mathrm{C}-3), 48.3$ $(2 \mathrm{C}-12), 52.5(2 \mathrm{C}-10), 52.8\left(2 \mathrm{OCH}_{3}\right), 54.8\left(2 \mathrm{OCH}_{3}\right), 57.9(2 \mathrm{C}-15), 63.3(2 \mathrm{C}-2), 67.1(2 \mathrm{C}-9), 102.4(2 \mathrm{C}-$ 14), 103.7 (2C-7), 129.7 (4 Ar-CH), 140.3 (2 Ar-C). MS (ESI $\left.{ }^{+}\right): m / z(\%) 649$ (40) [M+ Na] $]^{+}, 627$ (100) $[\mathrm{M}+\mathrm{H}]^{+}$. HRMS $\left(\mathrm{ESI}^{+}\right)$: calcd. for $[\mathrm{M}+\mathrm{H}]^{+}$627.4333, found 627.4329.

\subsection{Biological assays}

\subsubsection{Anti-HIV activity}

The cell cultures of MT- 4 and CEM-SS cells were maintained at $37{ }^{\circ} \mathrm{C}$ in a $5 \% \mathrm{CO}_{2}$ atmosphere in RPMI 1640 medium supplemented with $10 \%$ foetal calf serum (FCS) containing penicillin, streptomycin and glutamine. MT-4 cells were infected with HIV-1 ${ }_{\text {IIIB }}$ isolate at 5 TCID $_{50}$ (Tissue Culture Infective Dose) and CEM-SS cells with HIV-1 LAI isolate at 20 TCID $_{50}$. After $30 \mathrm{~min}$ of adsorption, the infected cells were washed to remove unadsorbed virus particles and resuspended in RPMI at the final concentration of $10^{5}$ cells $/ \mathrm{mL}$, in the presence of various dilutions of the test compounds. After 5 days, virus production was measured as already described [16]. The 50\% inhibitory concentration $\left(\mathrm{IC}_{50}\right)$ was derived from the computer-generated median effect plot of the dose-effect data [17]. The cytotoxicity of the drugs was evaluated in parallel by incubating uninfected cells in the presence of ranging concentrations of anti-viral products. Cell viability was measured by means of a colorimetric reaction based on the capacity of mitochondrial dehydrogenase of living cells to reduce 3-(4,5-dimethylthiazol-2-yl)-2,5diphenyltetrazolium bromide (MTT) into formazan, the quantity of which was determined by the optical density at $540 \mathrm{~nm}$ [18]. The 50\% cytotoxic concentration (CC50) was calculated with the program used for the determination of $\mathrm{IC}_{50}$. The CEM-SS cells were obtained from P. Nara through the AIDS Research and Reference Reagent Program, Division of AIDS, NIAID, NIH.

\subsubsection{Anti-tumoral activity}

The cell cultures were maintained at $37{ }^{\circ} \mathrm{C}$ under $5 \% \mathrm{CO}_{2}$ atmosphere for $72 \mathrm{~h}$, in either RPMI 1640 medium (cell lines: SF268, HL60, MCF7, MCF7R, HCT116, A549, PC3, OV3) or DMEM-Glutamax medium (cell lines: KB, HepG2), supplemented in both cases with $10 \%$ foetal calf serum (FCS) containing penicillin, streptomycin, fungizon and glutamine. Cells were plated at a density of 500-800 cells in $200 \mu \mathrm{L}$ culture medium in each well of 96-well microplates and allowed to adhere for $24 \mathrm{~h}$ before treatment with test drugs in DMSO solution (final concentration: $10^{-5} \mathrm{M}$ ). Cell viability was assayed after $3 \mathrm{~h}$ drug exposure with tetrazolium compound [3-(4,5-dimethylthiazol-2-yl)-5-(3carboxymethoxyphenyl)-2-(4-sulphophenyl)-2 $H$-tetrazolium salt, MTS, Promega Corp.] using the Cell 
Titer 96 Aqueous One Solution Cell Proliferation Assay kit (Promega Corp) according to the manufacturer's instructions. Absorbance was measured at $490 \mathrm{~nm}$ with a microplate reader.

\section{Acknowledgements}

The authors thank R. Egrot for the 2D HMBC NMR spectra, B. Légeret for the mass spectra (MS and HRMS), G. Laumond for the anti-HIV assays, and the Centre National de la Recherche Scientifique (CNRS), the Agence Nationale de Recherche sur le Sida (ANRS) and the Plan Auvergne Cancer (PAC) for financial supports.

\section{References}

[1] (a)E. Kimura, Tetrahedron 48 (1992), pp. 6175-6217.

(b)X. Liang and P.J. Sadler, Chem. Soc. Rev. 33 (2004), pp. 246-266.

[2] (a)E. De Clercq, N. Yamamoto, R. Pauwels, J. Balzarini, M. Witvrouw, K. De Vreese, Z. Debyser, B. Rosenwirth, P. Peichl, R. Datema, D. Thornton, R. Skerlj, F. Gaul, S. Padmanabhan, G. Bridger, G. Henson and M. Abrams, Antimicrob. Agents Chemother. (1994), pp. 668-674.

(b)G.J. Bridger, R.T. Skerlj, D. Thornton, S. Padmanabhan, S.A. Martellucci, G.W. Henson, M.J. Abrams, N. Yamamoto, K. De Vreese, R. Pauwels and E. De Clercq, J. Med. Chem. 38 (1995), pp. 366-378.

(c)H.C. Joao, K. De Vreese, R. Pauwels, E. De Clercq, G.W. Henson and G.J. Bridger, J. Med. Chem. 38 (1995), pp. $3865-$ 3873.

(d)G.J. Bridger, R.T. Skerlj, S. Padmanabhan, S.A. Martellucci, G.W. Henson, M.J. Abrams, H.C. Joao, M. Witvrouw, K. De Vreese, R. Pauwels and E. De Clercq, J. Med. Chem. 39 (1996), pp. 109-119.

[3] (a)G.A. Donzella, D. Schols, S.W. Lin, J.A. Esté, K.A. Nagashima, P.J. Maddon, G.P. Allaway, T.P. Sakmar, G. Henson, E. De Clercq and J.P. Moore, Nat. Med. 4 (1998), pp. 72-77.

(b)J.A. Esté, C. Cabrera, E. De Clercq, S. Struyf, J. Van Damme, G. Bridger, R.T. Skerlj, M.J. Abrams, G. Henson, A. Gutierrez, B. Clotet and D. Schols, Mol. Pharmacol. 55 (1999), pp. 67-73.

(c)G.J. Bridger, R.T. Skerlj, S. Padmanabhan, S.A. Martellucci, G.W. Henson, S. Struyf, M. Witvrouw, D. Schols and E. De Clercq, J. Med. Chem. 42 (1999), pp. 3971-3981.

(d)H.F. Egberink, E. De Clercq, A.L.W. Van Vliet, J. Balzarini, G.J. Bridger, G. Henson, M.C. Horzinek and D. Schols, J. Virol. 73 (1999), pp. 6346-6352.

(e)E. De Clercq, Mol. Pharmacol. 57 (2000), pp. 833-839.

[4] (a)B. Labrosse, A. Brelot, N. Heveker, N. Sol, D. Schols, E. De Clercq and M. Alizon, J. Virol. 72 (1998), pp. 6381-6388.

(b)L.O. Gerlach, R.T. Skerlj, G.J. Bridger and T.W. Schwartz, J. Biol. Chem. 276 (2001), pp. 14153-14160.

(c)S.P. Fricker, V. Anastassov, J. Cox, M.C. Darkes, O. Grujic, S.R. Idzan, J. Labrecque, G. Lau, R.M. Mosi, K.L. Nelson, L. Qin, Z. Santucci and R.S. Wong, Biochem. Pharmacol. 72 (2006), pp. 588-596.

[5] O. Wallace, Chem. Br. (2000), pp. 38-40.

[6] (a)A. Scozzafava, A. Mastrolorenzo and C.T. Supuran, J. Enzyme Inhib. Med. Chem. 17 (2002), pp. 69-76.

(b)A. Khan, J. Greenman and S.J. Archibald, Curr. Med. Chem. 14 (2007), pp. 2257-2277.

[7] (a)E. De Clercq, Nat. Rev. Drug Discov. 2 (2003), pp. 581-587.

(b)E. De Clercq, Mini Rev. Med. Chem. 5 (2005), pp. 805-824 and references cited therein. Full Text via CrossRef | View Record in Scopus | Cited By in Scopus (32)

[8]

(a)J.B. Rubin, A.L. Kung, R.S. Klein, J.A. Chan, Y.P. Sun, K. Schmidt, M.W. Kieran, A.D. Luster and R.A. Segal, Proc. Natl. Acad. Sci. U.S.A. 100 (2003), pp. 13513-13518.

(b)F. Marchesi, P. Monti, B.E. Leone, A. Zerbi, A. Vecchi, L. Piemonti, A. Mantovani and P. Allavena, Cancer Res. 64 (2004), pp. 8420-8427.

(c)M.C.P. Smith, K.E. Luker, J.R. Garbow, J.L. Prior, E. Jackson, D. Piwnica-Worms and G.D. Luker, Cancer Res. 64 (2004), pp. 8604-8612.

(d)H. Kajiyama, K. Shibata, M. Terauchi, K. Ino, A. Nawa and F. Kikkawa, Int. J. Cancer 122 (2008), pp. 91-99.

[9] (a)A. Larochelle, A. Krouse, M. Metzger, D. Orlic, R.E. Donahue, S. Fricker, G. Bridger, C.E. Dunbar and P. Hematti, Blood 107 (2006), pp. 3772-3778.

(b)S.G. Holtan, L.F. Porrata, I.N.M. Micallef, D.J. Padley, D.J. Inwards, S.A. Ansell, P.B. Johnston, D.A. Gastineau and S.N. Markovic, Clin. Lymphoma Myeloma (2007), pp. 315-318.

(c)J.L. Liesveld, J. Bechelli, K. Rosell, C. Lu, G. Bridger, G. Phillips II and C.N. Abboud, Leuk. Res. 31 (2007), pp. 15531563.

(d)A.F. Cashen, B. Nervi and J. DiPersio, Future Oncol. 3 (2007), pp. 19-27.

(e)G. Calandra, J. McCarty, J. McGuirk, G. Tricot, S.-A. Crocker, K. Badel, B. Grove, A. Dye and G. Bridger, Bone Marrow Transplant. 41 (2008), pp. 331-338.

[10] (a)S. Brandès, C. Gros, F. Denat, P. Pullumbi and R. Guilard, Bull. Soc. Chim. 133 (1996), pp. 65-73.

(b)D. Guillaume and G.R. Marshall, Synth. Commun. 28 (1998), pp. 2903-2906.

(c)M. Le Baccon, F. Chuburu, L. Toupet, H. Handel, M. Soibinet, I. Déchamps-Olivier, J.-P. Barbier and M. Aplincourt, New J. Chem. 25 (2001), pp. 1168-1174.

(d)M. Achmatowicz and L.S. Hegedus, J. Org. Chem. 68 (2003), pp. 6435-6436.

(e)F. Boschetti, F. Denat, E. Espinosa, A. Tabard, Y. Dory and R. Guilard, J. Org. Chem. 70 (2005), pp. 7042-7053. 
[11] (a)A.-G. Valade, D. Dugat, G. Jeminet, J. Royer and H.-P. Husson, Eur. J. Org. Chem. (2001), pp. $2041-2053$.

(b)Y. Pointud, A.-G. Valade, C. Pointon, D. Dugat, G. Jeminet and J.-L. Beltran, Supramol. Chem. 15 (2003), pp. $261-269$.

(c)A.-G. Valade, D. Dugat, B. Combourieu and J. Guyot, Tetrahedron 61 (2005), pp. 5641-5653.

[12] (a)R. Affani and D. Dugat, Synth. Commun. 37 (2007), pp. 3729-3740.

(b)R. Affani, P. Auzeloux, J.-C. Madelmont and D. Dugat, Eur. J. Org. Chem. (2008), pp. 2039-2048.

[13] Diketal dilactams, prepared in two steps from $\beta$-aminoalcohols, were isolated (i) in chiral series 1 and 2 , in three diastereoisomeric forms: an unsymmetrical trans-OMe isomer b, and two cis-OMe isomers a and c of C2 symmetry, a (minor isomer) and c (major isomer) differing in the trans or cis relationship of the OMe and R groups, and (ii) in achiral series 3 , under two diastereoisomeric forms $\mathrm{b}$ and $\mathrm{c}$ which both possess some symmetry (center in b, C2 axis in c)

[14] (a)J.-L. Pierre, H. Handel and R. Perraud, Tetrahedron 31 (1975), pp. 2795-2798 and references cited therein.

(b)E.C. Ashby and J.R. Boone, J. Am. Chem. Soc. 98 (1976), pp. 5524-5531.

(c)K.E. Wiegers and S.G. Smith, J. Org. Chem. 43 (1978), pp. 1126-1131.

[15] Evaluation made by the Biological Screening Platform, ICSN, UPR CNRS 2301, 91198 Gif-sur-Yvette, France.

[16] C. Moog, A. Wick, P. Le Ber, A. Kirn and A.-M. Aubertin, Antiviral Res. 24 (1994), pp. 275-288.

[17] J. Chou and T.C. Chou, Elsevier-Biosoft, Elsevier Science, Cambridge, U.K. (1985).

[18] T.J. Mosmann, Immunol. Methods 65 (1983), pp. 55-63. 